\title{
Deep probing of the photospheric sunspot penumbra: no evidence of field-free gaps
}

\author{
J. M. Borrero ${ }^{1}$, A. Asensio Ramos ${ }^{2,3}$, M. Collados ${ }^{2,3}$, R. Schlichenmaier ${ }^{1}$, H. Balthasar ${ }^{4}$, M. Franz ${ }^{1}$, R. Rezaei ${ }^{1,2,3}$, \\ C. Kiess ${ }^{1}$, D. Orozco Suárez ${ }^{2,3}$, A. Pastor ${ }^{2,3}$, T. Berkefeld ${ }^{1}$, O. von der Lühe ${ }^{1}$, D. Schmidt ${ }^{1}$, W. Schmidt ${ }^{1}$, \\ M. Sigwarth ${ }^{1}$, D. Soltau ${ }^{1}$, R. Volkmer ${ }^{1}$, T. Waldmann ${ }^{1}$, C. Denker ${ }^{4}$, A. Hofmann ${ }^{4}$, J. Staude ${ }^{4}$, K. G. Strassmeier ${ }^{4}$, \\ A. Feller ${ }^{5}$, A. Lagg $^{5}$, S. K. Solanki ${ }^{5,8}$, M. Sobotka ${ }^{7}$, and H. Nicklas ${ }^{6}$ \\ 1 Kiepenheuer-Institut für Sonnenphysik, Schöneckstr. 6, 79110 Freiburg, Germany \\ e-mail: borrero@leibniz-kis.de \\ 2 Instituto de Astrofísica de Canarias, Avd. Vía Láctea s/n, 38205 La Laguna, Spain \\ 3 Departamento de Astrofísica, Universidad de La Laguna, 38205 La Laguna, Tenerife, Spain \\ ${ }^{4}$ Leibniz Institute for Astrophysics Potsdam, An der Sternwarte 16, 14482 Potsdam, Germany \\ 5 Max Planck Institut for Solar System Reesearch, Justus-von-Liebig-Weg 3, 37077 Göttingen, Germany \\ ${ }_{7}^{6}$ Georg-August-Universität Göttingen, Faculty of Physics, Friedrich-Hund-Platz 1, 37077 Göttingen, Germany \\ 7 Astronomical Institute, Academy of Sciences of the Czech Republic, Fričova 298, 25165 Ondřejov, Czech Republic \\ 8 School of Space Research, Kyung Hee University, Yongin, 446-701 Gyeonggi, Republic of Korea \\ Received 15 February 2016 / Accepted 1 August 2016
}

\begin{abstract}
Context. Some models for the topology of the magnetic field in sunspot penumbrae predict regions free of magnetic fields or with only dynamically weak fields in the deep photosphere.

Aims. We aim to confirm or refute the existence of weak-field regions in the deepest photospheric layers of the penumbra.

Methods. We investigated the magnetic field at $\log \tau_{5}=0$ is by inverting spectropolarimetric data of two different sunspots located very close to disk center with a spatial resolution of approximately $0.4-0.45^{\prime \prime}$. The data have been recorded using the GRIS instrument attached to the $1.5-\mathrm{m}$ solar telescope GREGOR at the El Teide observatory. The data include three Fe I lines around $1565 \mathrm{~nm}$, whose sensitivity to the magnetic field peaks half a pressure scale height deeper than the sensitivity of the widely used Fe I spectral line pair at $630 \mathrm{~nm}$. Before the inversion, the data were corrected for the effects of scattered light using a deconvolution method with several point spread functions.

Results. At $\log \tau_{5}=0$ we find no evidence of regions with dynamically weak ( $B<500$ Gauss) magnetic fields in sunspot penumbrae. This result is much more reliable than previous investigations made on Fe I lines at $630 \mathrm{~nm}$. Moreover, the result is independent of the number of nodes employed in the inversion, is independent of the point spread function used to deconvolve the data, and does not depend on the amount of stray light (i.e., wide-angle scattered light) considered.
\end{abstract}

Key words. sunspots - Sun: infrared - Sun: photosphere - Sun: magnetic fields

\section{Introduction}

The past decade has been witness to an unprecedented advance in our knowledge of sunspot penumbrae. Owing to the improvement in instrumentation, data analysis methods, and realism of numerical simulations, a unified picture of the topology of penumbral magnetic and velocity fields has begun to emerge. The foundations of this picture rest on the so-called spine and intraspine structure of the sunspot penumbra, first mentioned by Lites et al. (1993), which means that regions of strong and somewhat vertical magnetic fields (the spines) alternate horizontally with regions of weaker and more inclined field lines that harbor the Evershed flow (the intraspines). At low spatial resolution $\left(\approx 1^{\prime \prime}\right)$ the intraspines are identified with penumbral filaments. At the same time, Solanki \& Montavon (1993) established that these two distinct components also interlace vertically, which explains the asymmetries in the observed circular polarization profiles (Stokes $V$ ). It was later found that the vertical and horizontal interlacing of these two components implies that the magnetic field in the spines wraps around the intraspines (Borrero et al. 2008), with the latter remaining unchanged at all radial distances from the sunspot center (Borrero et al. 2005, 2006; Tiwari et al. 2013), and the former being the extension of the umbral field into the penumbra (Tiwari et al. 2015). It has also been confirmed that the Evershed flow can reach supersonic and super-Alvénic values, not only on the outer penumbra (Borrero et al. 2005; van Noort et al. 2013), but also close to the umbra (del Toro Iniesta et al. 2001; Bellot Rubio et al. 2004), and has a strong upflowing component at the inner penumbra that becomes a downflowing component at larger radial distances (Franz \& Schlichenmaier 2009, 2013; Tiwari et al. 2013). Finally, there is strong evidence of an additional velocity field component in intraspines that appears as convective upflows along the center of the intraspines and changes into downflows at the filament edges (Zakharov et al. 2008; Joshi et al. 2011; Scharmer et al. 2011; Tiwari et al. 2013). These downflows seem capable of dragging the magnetic field lines and turning them back into the solar surface (Ruiz Cobo \& Asensio Ramos 2013; Scharmer et al. 2013).

In spite of this emerging unified picture, several controversies persist. One of them pertains to the strength of the 
convective upflows and downflows at the intraspine centers and edges, respectively. Tiwari et al. (2013), Esteban Pozuelo et al. (2015) found an average speed for this convective velocity pattern of about $200 \mathrm{~m} \mathrm{~s}^{-1}$. Although they are ubiquitous, their strength does not seem capable of sustaining the radiative cooling of the penumbra, which amounts to about $70 \%$ of the quietSun brightness. However, Scharmer et al. (2013) found an rms convective velocity of $1.2 \mathrm{~km} \mathrm{~s}^{-1}$ at the intraspine centers or edges, which is strong enough to explain the penumbral brightness. The latter result agrees well with numerical simulations of sunspot penumbrae (Rempel 2012). On the other hand, the pattern of upflows and downflows at the heads and tails, respectively, of the penumbral intraspines is easily discernible (Franz \& Schlichenmaier 2009, 2013; Ichimoto 2010) and harbors plasma flows of several $\mathrm{km} \mathrm{s}^{-1}$, but they occupy only a small fraction of the penumbral area. Which of these two convective modes accounts for the energy transfer in the penumbra is unclear from an observational point of view, although the scale is starting to tip in favor of the first.

Another remaining controversy concerns the strength of the magnetic field inside intraspines, where convection takes place. Scharmer \& Spruit (2006) and Spruit \& Scharmer (2006) originally proposed that intraspines were field-free, thereby coining the term field-free gap. However, most observational evidence points toward a magnetic field strength of at least $1 \mathrm{kG}$ (Borrero et al. 2008; Borrero \& Solanki 2010; Puschmann et al. 2010; Tiwari et al. 2013, 2015). Three-dimensional magnetohydrodynamic simulations of penumbral fine structure also yield magnetic field values of about $1-1.5 \mathrm{kG}$ inside penumbral intraspines (Rempel 2012) regardless of the boundary conditions and grid resolution. Spruit et al. (2010) interpreted the striations seen perpendicular to the penumbral filaments in high-resolution continuum images as a consequence of fluting instability, and established an upper limit of $B \leq 300$ Gauss for the magnetic field inside intraspines. This redefines field-free to mean instead dynamically weak magnetic fields, where the magnetic pressure is lower than the kinematic pressure. We note, however, that this interpretation has been challenged by Bharti et al. (2012), who argued that the same striations can be produced by the sideways swaying motions of the intraspines even if these harbor strong magnetic fields ( $B \geq 1000$ Gauss).

The limited observational evidence in favor of strong convective motions perpendicular to the penumbral filaments and the almost complete lack of evidence for weak magnetic fields in penumbral intraspines has been traditionally ascribed to (a) the insufficient spatial resolution of the spectropolarimetric observations (see Sect. 3.2 Scharmer \& Henriques 2012); (b) the smearing effects of stray light that are incorrectly dealt with by two-component inversions employing variable filling factors (see Sect. 2.2 in Scharmer et al. 2013); and (c) the impossibility of probing layers located deep enough to detect them (see Sect. 5.4 in Spruit et al. 2010). In this work we address these questions by employing spectropolarimetric observations of the Fe I spectral lines at $1565 \mathrm{~nm}$ recorded with the GRIS instrument at the GREGOR telescope. The spatial resolution is comparable to that of the Hinode/SP instrument and 2.5 times better than the resolution obtained by previous investigations that were carried out on these spectral lines. In addition, the lines observed by GRIS are much more sensitive to magnetic fields at the continuum-forming layer (i.e., $\log \tau_{5}=0$ ) than their counterparts at $630 \mathrm{~nm}$. Finally, we account for the stray light within the instrument by deconvolving the observations, for which we employ a principal component analysis (PCA) and different point spread functions (PSFs). We expect that with these new data and analysis techniques we are able to settle, in either direction, the dispute about the strength of the magnetic field in penumbral intraspines (e.g., filaments). A study of the convective velocity field will be presented elsewhere.

\section{Observations}

The observations employed in this work were taken with the 1.5-m GREGOR telescope (Schmidt et al. 2012) located at the Spanish observatory of El Teide. Our targets were two active regions: NOAA 12045 and the leading spot in NOAA 12049. They were observed on April 24, 2014 between UT 9:56 and 10:10, and on May 3, 2014 between UT 14:05 and 14:26.

The GREGOR Infrared Spectrograph (GRIS; Collados et al. 2012), which is coupled to the Tenerife Infrared Polarimeter (TIP2; Collados 2007), was used to record the Stokes vector $\boldsymbol{I}^{\text {obs }}(\lambda)=(I, Q, U, V)$ across a $4 \mathrm{~nm}$ wide wavelength region around $1565 \mathrm{~nm}$ and with a wavelength sampling of $\delta_{\lambda} \approx$ $40 \mathrm{m \AA}$ pixel $^{-1}$. This wavelength region was therefore sampled with about 1000 spectral points, out of which we selected a $2.4 \mathrm{~nm}$ wide region with $N_{\lambda}=600$ spectral points that includes three Fe I spectral lines (see Table 1). The large Landé factors and wavelengths of these lines ensure a high sensitivity to the magnetic field. In addition, the sensitivity of these spectral lines to the different physical parameters, in particular the magnetic field strength, peaks at an optical depth five times larger than in the case of the widely used Fe I lines at $630 \mathrm{~nm}$. The reason is that the $\mathrm{H}^{-}$opacity has a minimum at $1640 \mathrm{~nm}$ (Chandrasekhar $\&$ Breen 1946), which makes the Sun more transparent at these wavelengths than in the visible range, and that these spectral lines have a high excitation potential, which requires high temperatures (i.e., deep photospheric layers) to populate the energy levels involved in the electronic transition. More details are provided in Sect. 5.2.

The effective Landé factors in Table 1 have been obtained under the assumption of LS coupling. This is valid for the Fe I spectral lines at $1564.8 \mathrm{~nm}$ and $1566.2 \mathrm{~nm}$. The spectral line Fe I at $1565.2 \mathrm{~nm}$ is better described under JK coupling. However, following Bellot Rubio et al. (2000), we consider it to be a normal Zeeman triplet with an effective Landé factor of $g_{\mathrm{eff}}=1.45$.

During the observing time the solar image rotated, as a consequence of GREGOR's altitude-azimuthal mount (Volkmer et al. 2012), by about $5.6^{\circ}$ and $14.7^{\circ}$ for NOAA 12045 and NOAA 12049 , respectively. This was sufficiently small to avoid heavily distorting the continuum images reconstructed from the individual slit positions. The GREGOR adaptive optics system (GAOS; Berkefeld et al. 2012) worked throughout the entire scans (see Sects. 4.1.2 and 4.2). We note that the Fried parameter $r_{0}$ scales as $\lambda^{6 / 5}$, and therefore the size of the isoplanatic patches is about 2.5 times larger at $1565 \mathrm{~nm}$ than at $630 \mathrm{~nm}$, thereby allowing the AO to perform much better (e.g., compensating for seeing and optical aberrations) at larger wavelengths. Normalized continuum intensity images from the observed active regions are presented in Fig. 1. The contrast of the continuum intensity in the granulation in these images (at $1565 \mathrm{~nm}$ ) is about $2.2 \%$. This value is equivalent to a $5.5 \%$ contrast at $630 \mathrm{~nm}$, which is lower than the $7.2 \%$ contrast seeing by Hinode/SP, thus indicating that the spatial resolution is slightly lower than that of Hinode/SP (i.e., 0.32"). To estimate it more accurately, we determined a cutoff frecuency of between 2 and $3 \operatorname{arcsec}^{-1}$ in the power spectra of the continuum intensity in the granulation, yielding a spatial resolution of about $0.4-0.45^{\prime \prime}$.

By correlating our images with simultaneous HMI/SDO fulldisk continuum images, we estimate that the sunspot centers 
Table 1. Atomic parameters of the observed spectral lines.

\begin{tabular}{cccccccc}
\hline \hline Ion & $\begin{array}{c}\lambda_{0}{ }^{a} \\
{[\AA]}\end{array}$ & $\begin{array}{c}\chi_{\text {low }}{ }^{a} \\
{[\mathrm{eV}]}\end{array}$ & $\log (g f)$ & Elec.conf $^{a}$ & $\sigma$ & $\alpha$ & $g_{\text {eff }}$ \\
\hline Fe I & 15648.515 & 5.426 & $-0.669^{b}$ & ${ }^{7} D_{1}-{ }^{7} D_{1}$ & $975^{b}$ & $0.229^{b}$ & 3.0 \\
Fe I & 15652.874 & 6.246 & $-0.095^{b}$ & ${ }^{7} D_{5}-{ }^{6} D_{4.5} 4 f[3.5]^{0}$ & $1427^{b}$ & $0.330^{b}$ & 1.45 \\
Fe I & 15662.018 & 5.830 & $0.190^{c}$ & ${ }^{5} F_{5}-{ }^{5} F_{4}$ & $1197^{c}$ & $0.240^{c}$ & 1.5 \\
\hline
\end{tabular}

Notes. $\lambda_{0}$ is the central laboratory wavelength of each spectral line. $\sigma$ and $\alpha$ represent the cross-section (in units of Bohr's radius squared $a_{0}^{2}$ ) and velocity parameter of the atom undergoing the transition, respectively, for collisions with neutral atoms under the ABO theory (Anstee \& O’Mara 1995; Barklem \& O’Mara 1997; Barklem et al. 1998). ${ }^{(a)}$ Values taken from Nave et al. (1994). ${ }^{(b)}$ Values taken from Borrero et al. (2003).

(c) Values taken from Bloomfield et al. (2007).

were located at coordinates $(x, y)=\left(125^{\prime \prime},-309^{\prime \prime}\right)$ and $(x, y)=$ $\left(73^{\prime \prime},-83^{\prime \prime}\right)$ (measured from disk center) for NOAA 12045 and NOAA 12049, respectively. These values correspond to heliocentric angles of $\Theta=20.5^{\circ}(\mu=0.936)$ and $\Theta=6.5^{\circ}$ $(\mu=0.993)$. The image scale was also estimated by correlating the images with HMI data, yielding $\delta_{x}=0.135^{\prime \prime}$ pixel $^{-1}$ and $\delta_{y}=0.136^{\prime \prime}$ pixels $^{-1}$ along the $x$ and $y$ axis, respectively. The sizes of the scanned regions are $60 \times 27 \operatorname{arcsec}^{2}$ (top panel in Fig. 1) and $53 \times 45 \operatorname{arcsec}^{2}$ (bottom panel in Fig. 1). The width of the spectrograph slit was set to $0.27^{\prime \prime}$ (i.e., twice the scanning step).

The data were treated with dark current substraction, flatfield correction, and fringe removal (see Franz et al. 2016, for more details) and were polarimetrically calibrated (see Collados et al., in prep.), yielding a noise level of about $\sigma_{q} \approx \sigma_{u} \approx \sigma_{v} \approx$ $10^{-3}$ in units of the quiet-Sun continuum intensity. These values were achieved with $30 \mathrm{~ms}$ exposures per accumulation and a total of five accumulations per modulation step. The data were wavelength calibrated under the assumption that the averaged quiet-Sun intensity spectral line profiles, obtained as the mean $I(\lambda)$ inside the blue rectangles in Fig. 1, are located at the central laboratory wavelength position $\lambda_{0}$ (see Table 1), but shifted by $-535 \mathrm{~m} \mathrm{~s}^{-1}$ to account for the convective blueshift in the observed spectral lines as determined from the Fourier Transform Spectrometer (FTS; Livingston \& Wallace 1991). After these standard calibrations, the data were corrected for spectral scattered light within the spectrograph (Sect. 3), and for the smearing effects introduced by the telescope PSF (Sect. 4.1).

\section{Spectral profile: veil correction}

Before analyzing the recorded data, we estimated the spectral profile of GRIS, for which we employed a similar procedure as described in Bianda et al. (1998), Allende Prieto et al. (2004), Cabrera Solana et al. (2007). We first obtained a GRIS-simulated average quiet-Sun intensity profile $I_{\mathrm{qs}}^{\mathrm{sim}}(\lambda)$ by convolving FTS data with a wavelength profile $t(\lambda)$ that mimics the effects of the spectral profile of GRIS:

$I_{\mathrm{qs}}^{\mathrm{sim}}(\lambda)=I_{\mathrm{fts}}(\lambda) * t(\lambda)$.

In principle, $t(\lambda)$ can be approximated by a Gaussian function $g(\lambda, \sigma)$, where $\sigma$ refers to the width of spectral profile. However, a Gaussian profile decays rapidly with wavelength, which means that the possible effect of extended wings in the spectral profile would be neglected. These wings can be interpreted as spectral scattered light or a spectral veil that mixes information from faraway wavelengths. As a first approximation, we can consider this spectral veil to be independent of wavelength and proportional to

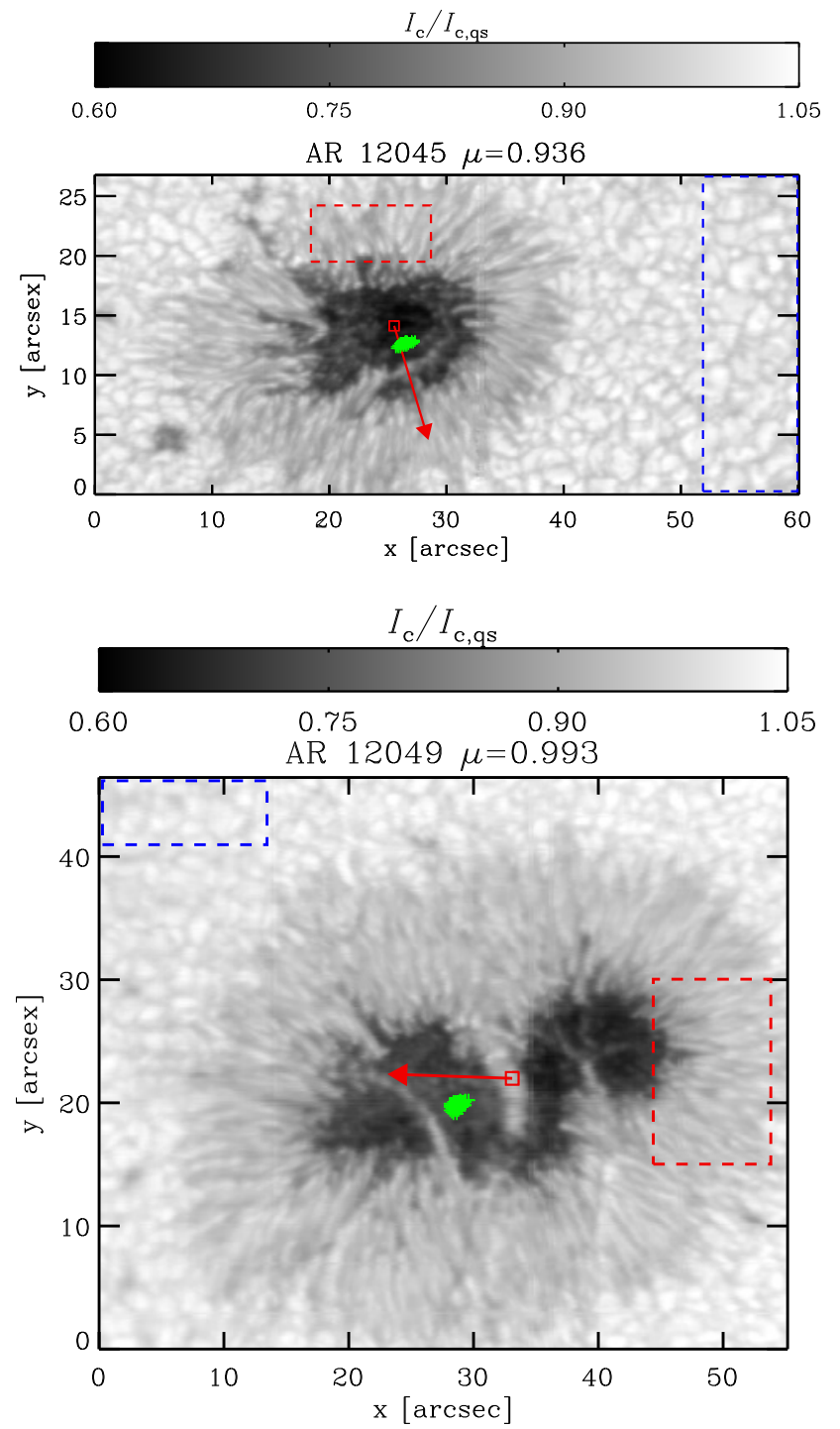

Fig. 1. Continuum intensity $I_{\mathrm{c}}$ (at $1565 \mathrm{~nm}$ ) of NOAA 12045 (top) and leading spot of NOAA 12049 (bottom) normalized to the quiet-Sun continuum $\left(I_{\mathrm{c}, \mathrm{qs}}\right)$ as observed with the GREGOR Infrared Spectrograph (GRIS) on April 24, 2014 and May 3, 2015, respectively. The red squares denote the center of each sunspot: $(x, y)=\left(125^{\prime \prime},-309^{\prime \prime}\right)$ for NOAA 12045 and $(x, y)=\left(73^{\prime \prime},-83^{\prime \prime}\right)$ for NOAA 12049 . The red arrow points toward the center of the solar disk. The areas enclosed by the blue dashed rectangles have been used for calibration purposes (e.g., to calculate the normalization factor $I_{\mathrm{c}, \mathrm{q}}$; see also Sect. 3), while the areas enclosed by the red dashed rectangles indicate the regions that have been analyzed in our work (see Sects. 4.3 and 5). The scanning direction (i.e., direction of movement of the spectrograph slit) was from top to bottom along the $y$-axis. 

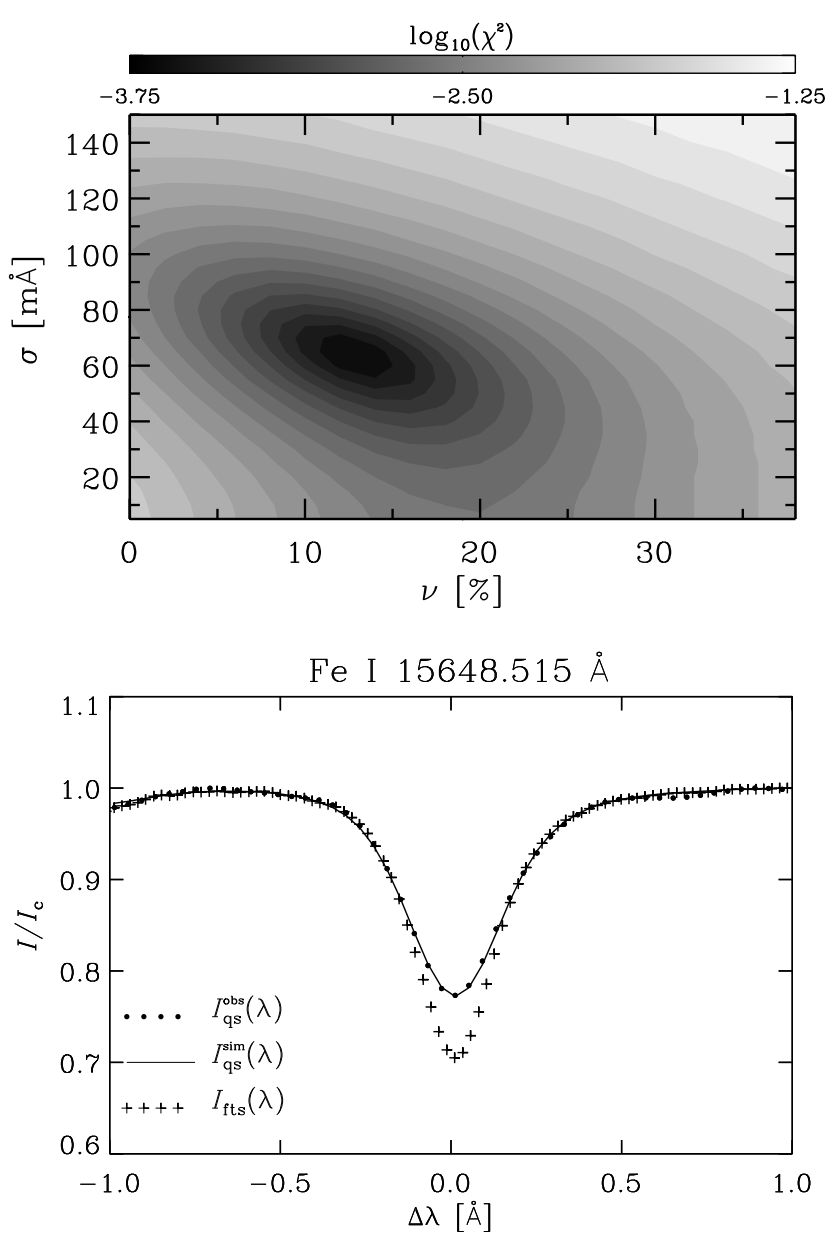

Fig. 2. Top panel: $\chi^{2}$-surface as a function of the parameters $v$ and $\sigma$ defining the spectrograph profile (Eq. (2)). This surface was obtained from comparing the observed $\left(I_{\mathrm{qs}}^{\mathrm{obs}}\right)$ and simulated $\left(I_{\mathrm{qs}}^{\mathrm{sim}}\right)$ quiet-Sun intensity profile of the Fe Iline at $1564.8 \mathrm{~nm}$. Bottom panel: FTS intensity profile of Fe I $1564.8 \mathrm{~nm} I_{\mathrm{fts}}$ (crosses), simulated average quiet-Sun intensity profile obtained after applying the spectrograph profile to FTS data $I_{\mathrm{qs}}^{\mathrm{sim}}$ (solid line), and observed average quiet-Sun intensity profile from GRIS data $I_{\mathrm{qs}}^{\mathrm{obs}}$ in NOAA 12049 (filled circles; see also blue dashed rectangles in Fig. 1).

the continuum intensity, in which case Eq. (1) becomes

$I_{\mathrm{qs}}^{\mathrm{sim}}(\lambda, \sigma, v)=(1-v) I_{\mathrm{fts}}(\lambda) * g(\lambda, \sigma)+v I_{\mathrm{c}, \mathrm{fts}}$,

where $I_{\mathrm{c}, \mathrm{fts}}=I_{\mathrm{fts}}\left(\lambda_{\mathrm{c}}\right)$ is the continuum intensity in FTS data and $v$ is the fraction of spectral scattered light. Next, we created an array of simulated averaged quiet-Sun intensity profiles through Eq. (2), $I_{\mathrm{qs}}^{\operatorname{sim}}(\lambda)$, employing different values of $\sigma$ and $v$. Each of these was then compared through a $\chi^{2}$ merit-function, with the observed (i.e., by GRIS) average quiet-Sun intensity profile $I_{\mathrm{qs}}^{\mathrm{obs}}(\lambda)$ that is obtained by averaging the intensity profiles in the quiet-Sun region denoted by the blue dashed rectangles in each map in Fig. 1:

$\chi^{2}(\sigma, v)=\sum_{k=1}^{N_{\lambda}^{\prime}}\left[I_{\mathrm{qs}}^{\mathrm{sim}}\left(\lambda_{k}, \sigma, v\right)-I_{\mathrm{qs}}^{\mathrm{obs}}\left(\lambda_{k}\right)\right]^{2}$,

where the index $k=1, \ldots, N_{\lambda}^{\prime}$ runs for all wavelengths observed by GRIS across a particular spectral line. We note that, since the spectral sampling of GRIS $\left(\approx 40 \mathrm{~m} \AA\right.$ pixel $\left.^{-1}\right)$ is coarser

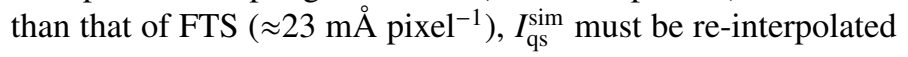

to the GRIS wavelength grid before Eq. (3) can be evaluated. Figure 2 (top panel) shows the $\chi^{2}$ surface as a function of $\sigma$ and $v$ that results from the comparison of $I_{\mathrm{qs}}^{\mathrm{sim}}(\lambda)$ and $I_{\mathrm{qs}}^{\mathrm{obs}}(\lambda)$ for the Fe I spectral line located at $1564.8 \mathrm{~nm}$ (see Table 1). The minimum of this surface is attained for $\sigma=70 \mathrm{~m} \AA$ and $v=0.12$ (12\% of the spectral veil). This value of $\sigma$ corresponds to a FWHM for the spectral transmission of $g(\lambda, \sigma)$ of approximately $165 \mathrm{~m} \AA$ (cf. Franz et al. 2016). The same values are obtained for the two sunspots in Fig. 1. Figure 2 (bottom panel) compares the original FTS intensity profile $I_{\mathrm{fts}}(\lambda)$ (crosses), the simulated quiet-Sun profile $I_{\mathrm{qs}}^{\mathrm{sim}}(\lambda)$ (solid lines) obtained through Eq. (2) with the aforementioned values of $\sigma$ and $v$, and the GRIS observed quiet-Sun profile $I_{\mathrm{qs}}^{\mathrm{obs}}(\lambda)$ in NOAA 12049 (filled circles). $I_{\mathrm{qs}}^{\mathrm{obs}}(\lambda)$ and $I_{\mathrm{qs}}^{\mathrm{sim}}(\lambda)$ are extremely similar, as guaranteed by the low $\chi^{2}$ value (top panel in Fig. 2).

With this, it is now possible to substract the effect of the spectral veil from the observed intensity profiles at every pixel $(x, y)$ in the entire field of view and obtain corrected profiles, $I^{\text {cor }}(x, y, \lambda)$, by simply applying

$I^{\mathrm{cor}}(x, y, \lambda)=[1-v]^{-1}\left[I^{\mathrm{obs}}(x, y, \lambda)-v I_{\mathrm{c}}^{\mathrm{obs}}(x, y)\right]$.

At this point a number of clarifications are in order. The first is that the correction for spectral veil only affects the intensity profiles since the continuum polarization is zero $Q_{\mathrm{c}}^{\mathrm{obs}}(x, y)=$ $U_{\mathrm{c}}^{\mathrm{obs}}(x, y)=V_{\mathrm{c}}^{\mathrm{obs}}(x, y)=0$ everywhere, which means that $Q^{\text {cor }}=Q^{\text {obs }}$ (and likewise for $U$ and $V$ ). In the following sections we no longer consider $\boldsymbol{I}^{\text {obs }}(x, y, \lambda)$, but only the veil-corrected Stokes vector $\boldsymbol{I}^{\mathrm{cor}}(x, y, \lambda)$. However, for the sake of simplicity, we continue to refer to it as $\boldsymbol{I}^{\mathrm{obs}}(x, y, \lambda)$.

Finally, it must be borne in mind that by applying Eq. (4) here we only correct for the spectral veil. The remaining spectrograph profile, namely the Gaussian profile $g(\lambda, \sigma)$ in Eq. (2) (with $\sigma=$ $70 \mathrm{m \AA}$ ), is considered at a later step in the analysis (Sect. 4.3).

\section{Analysis}

\subsection{Spatial point spread function}

Seeing, scattered light, and diffraction effects cause the observed Stokes vector to differ from the vector emitted at a point $(x, y)$ on the solar surface. These effects can be quantified through the PSF of the optical system $P(x, y)$, which smears out the original signal:

$\boldsymbol{I}^{\mathrm{obs}}(x, y, \lambda)=\iint \boldsymbol{I}^{\mathrm{sun}}\left(x^{\prime}, y^{\prime}, \lambda\right) P\left(x-x^{\prime}, y-y^{\prime}\right) \mathrm{d} x^{\prime} \mathrm{d} y^{\prime}$.

This equation indicates that some percentage of the signal from points $\left(x^{\prime}, y^{\prime}\right)$ with $x^{\prime} \neq x$ and $y^{\prime} \neq y$, will contaminate the Stokes vector at the location $(x, y)$. Cleaning the observed signal of this effect has been traditionally limited to data from space-borne instrumentation where the optical system has a well-defined $P(x, y)$ function that can be calculated, sometimes even analytically, for all types of instruments (Danilovic et al. 2008; Wedemeyer-Böhm 2008; Mathew et al. 2009; Asensio Ramos \& López Ariste 2010; Yeo et al. 2014). From the ground the situation becomes more complicated, as the time-varying seeing imposes strong limits as to how fast the observations must be carried out in order to be corrected. In this case, $P(x, y)$ must be determined empirically by reconstruction techniques such as speckle-reconstruction (Keller \& von der Luehe 1992), multi-object multi-frame blind deconvolution (Löfdahl 2002; van Noort et al. 2005), or phase diversity (Paxman et al. 1996). 
For these reasons, the procedure has been limited for groundbased observations to high-throughput filter-based spectropolarimeters (Scharmer et al. 2008; Bello González \& Kneer 2008; Del Moro et al. 2010; Martínez Pillet et al. 2011). While these techniques should in principle deliver diffraction-limited observations, recently Scharmer \& Henriques (2012) and Löfdahl \& Scharmer (2012) have argued that high-altitude seeing remains uncorrected.

High-order adaptive optics allow us today to obtain spectropolarimetric data from slit-based instruments that are stable enough during acquisition, so that we can attempt to decontaminate $\boldsymbol{I}^{\text {obs }}$ and retrieve $\boldsymbol{I}^{\text {sun }}$ (Eq. (5)). The problem remains, however, how to obtain a PSF that represents the optical system, including seeing, in ground-based long-slit spectrographs (Beck et al. 2011). Although the AO-system can correct for most of the low- and mid-order optical aberrations, it cannot correct for all of them. Moreover, the correction weakens for regions away from the AO lockpoint. Consequently, the observations are never completely diffraction limited, which prevents a good knowledge of $P(x, y)$. We therefore resorted to indirect means to obtain a meaningful PSF that can be employed to isolate the solar signal. We first assumed for simplicity that the PSF can be described by two different Gaussian functions corresponding to narrow, $n$, and wide, $w$, angle contributions,

$P(x, y)=p_{n} g_{n}\left(x, y, \sigma_{n}\right)+p_{w} g_{w}\left(x, y, \sigma_{w}\right)$,

where $\sigma_{n}$ and $\sigma_{w}$ correspond to the distances, in seconds of arc, in which each Gaussian contributes with $\approx 68.2 \%$ of its power. $p_{n}$ and $p_{w}$ denote the relative contribution from narrow and wide angles, respectively. We note that $P(x, y), g_{w}$, and $g_{n}$ are normalized to unity, and that $p_{w}+p_{n}=1$.

\subsubsection{Estimation of $p_{w}$ and $\sigma_{w}$}

Sunspots close to disk center always have a region, located within the umbra, where the magnetic field is aligned with the observer line of sight. To locate these regions within the observed FOV, we selected those pixels in Fig. 1 where $I_{\mathrm{c}} / I_{\mathrm{c}, \mathrm{qs}}<$ 0.7 and where the maximum of the total linear polarization $\max \left|\left(Q^{2}+U^{2}\right)^{1 / 2}\right|<5 \times 10^{-3}$. In total there are about 60 pixels inside the umbra of each sunspot that fulfill these conditions. They are marked in Fig. 1 with green crosses. We refer to the location of these pixels as $\left(x^{*}, y^{*}\right)$.

We also recall that whenever a magnetic field is aligned with the observer line of sight, the intensity profile $I(\lambda)$ of a normal Zeeman triplet $\left(J_{l}=0 \rightarrow J_{u}=1\right.$ or vice versa) presents two (and only two) distinct absorption features according to the selection rule $\Delta M= \pm 1$ for the Zeeman effect (del Toro Iniesta 2003). Each of these two absorption features is shifted with respect to the central wavelength by an amount that is proportional to $\propto\left(g_{\text {eff }} B \Delta M\right)$, where $B$ is the modulus of the magnetic field. When the separation between these two components is sufficiently large, they appear as two unblended spectral lines, and therefore the observed intensity at the central wavelength must be very close to the continuum intensity: $I\left(\lambda^{\dagger}\right) \approx I_{\mathrm{c}}=I\left(\lambda_{\mathrm{c}}\right)$. To ensure that these conditions are met, we used the intensity profile of Fe I $1564.8 \mathrm{~nm}$ because this is the spectral line in our observations with the largest Landé factor (see Table 1) and the only one featuring a normal Zeeman triplet. We note that $\lambda^{\dagger}$ does not necessarily correspond to the central laboratory wavelength $\lambda_{0}$. Instead it is defined as the wavelength located half the way between the two absorption features with $\Delta M= \pm 1$.

Interestingly, the profiles selected above and located at $\left(x^{*}, y^{*}\right)$ do exhibit a small absorption feature at $\lambda^{\dagger}$. As the magnetic field
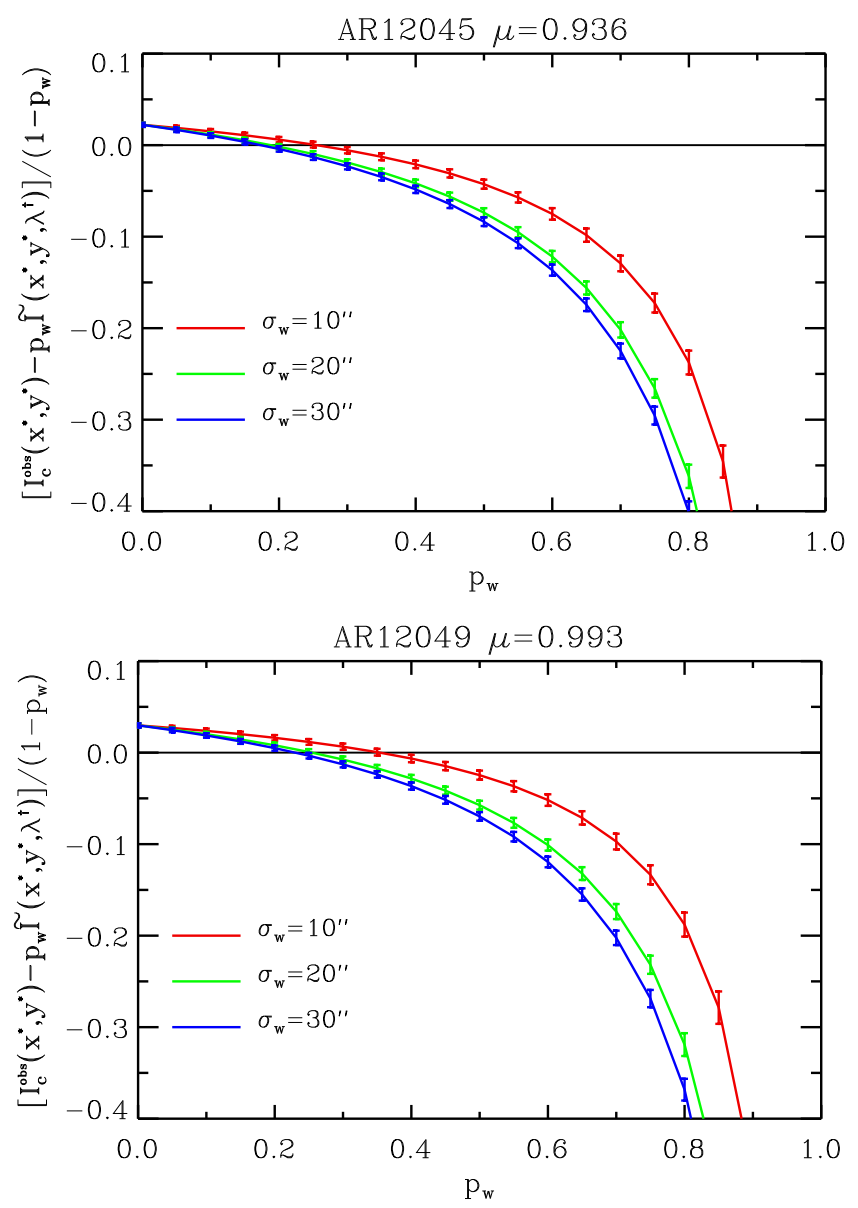

Fig. 3. Determination of the amount of stray light $p_{w}$ (wide-angle contribution) by finding the value of $p_{w}$ for which the intensity at central wavelength $\lambda^{\dagger}$ in those pixels $\left(x^{*}, y^{*}\right)$, where the magnetic field is aligned with the observer line of sight is equal to the continuum intensity. This occurs for $p_{w}=0.2-0.3$ depending on the width $\sigma_{w}$ of the Gaussian employed to mimic the effects of the stray light. The vertical error bars refer to the standard deviation in the determination of $p_{w}$ using each of the pixels in $\left(x^{*}, y^{*}\right)$.

is mostly aligned to the observer line of sight in these pixels, the absorption feature at $\lambda^{\dagger}$ is unlikely to be produced by the magnetic field (unshifted $\Delta M=0$ component of the Zeeman pattern). Instead, it must arise from an absorption profile unaffected by the magnetic field (i.e., penumbra and quiet Sun surrounding both sunspots). To determine where this contribution comes from and how much there is, we calculated a new intensity profile at each of the selected pixels $\widetilde{I}\left(x^{*}, y^{*}\right)$ in the following way:

$\widetilde{I}\left(x^{*}, y^{*}, \lambda\right)=\iint g_{w}\left(x-x^{*}, y-y^{*}, \sigma_{w}\right) I^{\mathrm{obs}}(x, y, \lambda) \mathrm{d} x \mathrm{~d} y$,

where $g_{w}$ was defined in Eq. (6). Next, we calculated the difference between the continuum intensity at the location $\left(x^{*}, y^{*}\right)$, that is, $I_{\mathrm{c}}^{\mathrm{obs}}\left(x^{*}, y^{*}\right)$, and $p_{w} \widetilde{I}\left(x^{*}, y^{*}, \lambda^{\dagger}\right)$, for different values of $p_{w}$ and $\sigma_{w}$. Since the bulk of the contribution to the absorption feature observed at $\lambda^{\dagger}$ is ascribed to the penumbra and granulation and these are located about $10^{\prime \prime}$ away from $\left(x^{*}, y^{*}\right)$, then $\sigma_{w} \geq 10^{\prime \prime}$. The results are presented in Fig. 3 for the two sunspots in our dataset. The intensity at the central wavelength position becomes comparable to the continuum intensity, $I^{\mathrm{obs}}\left(\lambda^{\dagger}\right) \approx I_{\mathrm{c}}^{\mathrm{obs}}$, for $p_{w} \approx 0.2-0.3$. This indicates that the 

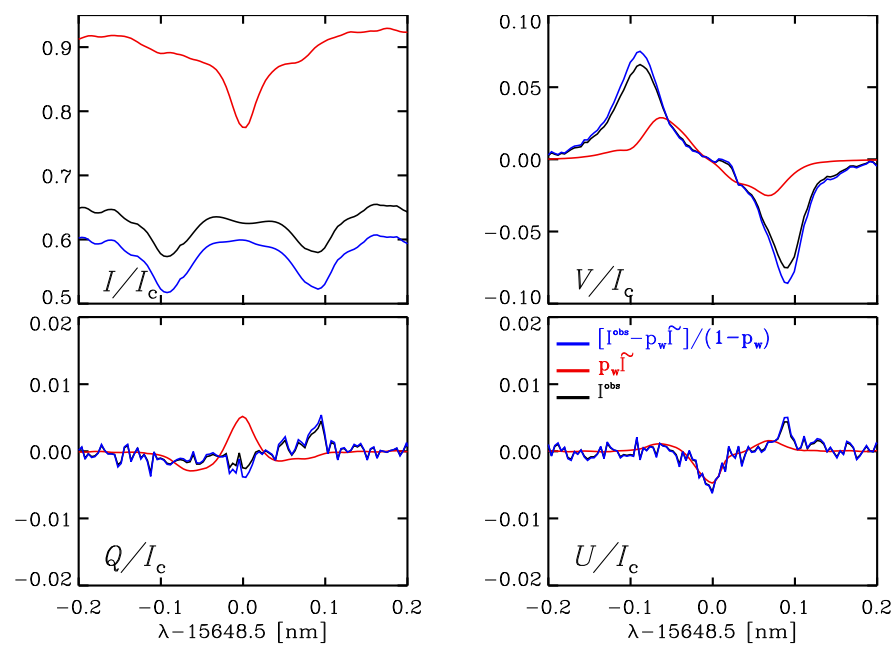

Fig. 4. Example of determining $\sigma_{w}$ and $p_{w}$ by removing the $\pi$-component in the intensity profile (Stokes $I$; top left panel) in a pixel in which the magnetic field is aligned with the observer line of sight Black lines represent the observed Stokes profiles $\boldsymbol{I}^{\text {obs }}$. Red lines show the wide-angle contribution (i.e., scattered light) from the surrounding penumbra and quiet Sun, $\tilde{\boldsymbol{I}}$. Blue lines represent the observed Stokes profiles after removing $20 \%$ of the wide-angle contribution. See text for details.

absorption feature seen at $\lambda^{\dagger}$ in the pixels in which the magnetic field is aligned with the observer line of sight, $\left(x^{*}, y^{*}\right)$ can be explained with a 20-30\% stray-light contamination from outside the umbra. Unfortunately, the exact distance cannot be reliably determined since the same values of $p_{w}$ are obtained for three different values of $\sigma_{w}$. As a compromise, we adopted $\sigma_{w}=20^{\prime \prime}$ and $p_{w}=0.2$. It should be noted, however, that the smaller $\sigma_{w}$, the larger $p_{w}$ to explain the absorption feature seen at $\lambda^{\dagger}$. The reason is that $\widetilde{I}\left(x^{*}, y^{*}, \lambda\right)$ does not include a strong absorption at $\lambda^{\dagger}$ for low values of $\sigma_{w}$ because $g_{w}$ (see Eq. (7)) mainly includes contributions from the neighborhood of $\left(x^{*}, y^{*}\right)$ (i.e., the sunspot umbra)

The procedure described above is illustrated for one of the pixels referred to as $\left(x^{*}, y^{*}\right)$ in Fig. 4. This figure depicts the observed Stokes profiles $\boldsymbol{I}^{\text {obs }}$ in black lines. Stokes $I$ (upper left panel) has a small absorption feature at a wavelength $\lambda^{\dagger} \approx$ $1564.85[\mathrm{~nm}]$ located in between the two $\sigma$-components of the Zeeman pattern. The very weak observed $Q$ (bottom left) and $U$ (bottom right) profiles, along with the strong circular polarization signals (Stokes $V$; top right) implies that the magnetic field in this pixel is mostly aligned with the observer line of sight. With red lines we show the contribution from the surrounding penumbra and quiet Sun $\tilde{\boldsymbol{I}}$ as calculated through Eq. (7) assuming that about $68 \%$ of the wide-angle scattered light comes from a distance around the considered pixel of $20^{\prime \prime}$ : $\sigma_{w}=20^{\prime \prime}$. Blue lines show the umbral profile in the same pixel after removing $p_{w} \tilde{\boldsymbol{I}}$ from $\boldsymbol{I}^{\mathrm{obs}}$ using $p_{w}=0.2$ and renormalizing using $\left(1-p_{w}\right)$. The resulting Stokes $I$ profile (blue lines in the top left panel) does no longer feature the absorption at $\lambda^{\dagger}$ (i.e., where the unshifted $\Delta M=0$ would appear). At this wavelength the observed intensity is the same as the continuum intensity.

It is important to mention at this point that the absorption feature seen at $\lambda^{\dagger}$ might also be caused by unidentified molecular blends that are common in the umbra at near-IR wavelengths, leftover fringes, and even by the magnetic field not being fully aligned with the observer line of sight. It might also appear as a combination of all the above. Because we have assumed only one origin, the values for $p_{w}$ obtained in this section are only to be considered as an upper limit of the real amount of wide-angle scattered light present in our observations.

\subsubsection{Estimation of $p_{n}$ and $\sigma_{n}$}

When $p_{w}$ is known, it is straightforward to determine $p_{n}$ as $p_{n}=1-p_{w}=0.8$. The width of the narrow-angle PSF, $\sigma_{n}$, is determined by performing a slit-scan of the pinhole array while it is inserted into the light-path at the third focal point along the optical path (i.e., before the spectrograph slit). By fitting the shape of the light curve at the pinhole discontinuity, a value of 3.2 pixels on the CCD was determined. Considering the values for the image scale given in Sect. 2, this yields $\sigma_{n}=0.18^{\prime \prime}$, corresponding to a FWHM of $0.43^{\prime \prime}$ and very similar to the spatial resolution estimated from the power spectrum of the granulation (Sect. 2). The theoretical diffraction-limited FHWM is $\approx 0.27^{\prime \prime}$, but this value accounts only for the primary mirror. Considering the effects of the spiders and central obscuration of the secondary mirror shows that the performance is still not ideal. While we did not investigate in detail the reason for this, we can point to several possible sources such as high-altitude seeing (Scharmer \& Henriques 2012; Löfdahl \& Scharmer 2012), width of the spectrograph slit, and a slightly off-focus spectrograph.

\subsection{PCA expansion and deconvolution}

After empirically estimating the PSF of the optical system $P(x, y)$ we can attempt to retrieve $\boldsymbol{I}^{\text {sun }}$ by deconvolving $\boldsymbol{I}^{\text {obs }}$ through Eq. (5). One possibility would be to deconvolve individual two-dimensional monochromatic images (e.g., $I^{\mathrm{obs}}\left(x, y, \lambda_{k}\right)$ for $k=1, \ldots, N_{\lambda}=600$ and likewise for $Q, U$, and $V$ ). However, this approach neglects the information contained in the wavelength dependence of the Stokes parameters. This is important because this information allows us to reduce the influence of the noise in the deconvolution process. Currently, two methods take advantage of this wavelength dependence. The first, referred to as spatially coupled inversions (van Noort 2012), uses the radiative transfer equation for polarized light to exploit the wavelength dependence of the Stokes vector during the deconvolution process (Riethmüller et al. 2013; van Noort et al. 2013; Lagg et al. 2014; Tiwari et al. 2015). This method has the advantage that is physically driven, but it requires deep modifications in existing inversion codes for the radiative transfer equation. The second method is based on the principal component analysis (PCA) of the data (Ruiz Cobo \& Asensio Ramos 2013; Quintero Noda et al. 2015). Although this is statistically driven, it has the advantage that it can be used in combination with any existing inversion codes for the radiative transfer equation without further modifications. Because of its simplicity, we chose the second method for our analysis. It must be borne in mind, however, that it remains to be proven that these two methods give the same results when applied to the same data.

Hence we followed Ruiz Cobo \& Asensio Ramos (2013) and Quintero Noda et al. (2015) and expanded each of the four components of the Stokes vector $\left(\boldsymbol{I}^{\mathrm{obs}}\right.$ and $\left.\boldsymbol{I}^{\text {sun }}\right)$ over the entire field of view in Fig. 1 in a set of orthonormal eigenvectors $\phi(\lambda)$ such 
that

$$
\begin{aligned}
I_{m}^{\mathrm{obs}}(x, y, \lambda) & =\sum_{n=1}^{N_{\lambda}} C_{m, n}^{\mathrm{obs}}(x, y) \phi_{m, n}(\lambda) \\
& \simeq \sum_{n=1}^{N_{m}^{\mathrm{pca}}} C_{m, n}^{\mathrm{obs}}(x, y) \phi_{m, n}(\lambda) . \\
I_{m}^{\mathrm{sun}}(x, y, \lambda) & =\sum_{n=1}^{N_{\lambda}} C_{m, n}^{\mathrm{sun}}(x, y) \phi_{m, n}(\lambda) \\
& \simeq \sum_{n=1}^{N_{m}^{\mathrm{pca}}} C_{m, n}^{\mathrm{sun}}(x, y) \phi_{m, n}(\lambda),
\end{aligned}
$$

where the index $m=1, . ., 4$ corresponds to any of the four components of the Stokes vector, $I, \ldots, V$, respectively, and the index $n=1, \ldots, N_{\lambda}$ runs for the total number of observed wavelengths (see Sect. 2) on the upper part of the above equations. The summation over index $n$ is truncated to $N_{m}^{\text {pca }}$, hence the approximate symbol, in the lower part of the equations. This is explained below. We note the implicit assumption that the same set of $n$-eigenvectors $\phi_{m, n}(\lambda)$ can be used to expand the $m$ th component of both the observed and solar Stokes vector. The PCA analysis provides a method to calculate the eigenvectors $\phi$ and eigenvalues $C$ by diagonalizing (through the singular value decomposition method) the matrix of the observed Stokes profiles (Quintero Noda et al. 2015) or, equivalently, the correlation matrix of the observed Stokes profiles (Skumanich \& López Ariste 2002; Casini et al. 2013). When these were obtained, we substituted Eqs. (8) and (9) into Eq. (5),

$$
\begin{aligned}
& \sum_{n=1}^{N_{\lambda}} C_{m, n}^{\mathrm{obs}}(x, y) \phi_{m, n}(\lambda)= \\
& \quad \sum_{n=1}^{N_{\lambda}}\left[\iint C_{m, n}^{\mathrm{sun}}\left(x^{\prime}, y^{\prime}\right) P\left(x-x^{\prime}, y-y^{\prime}\right) \mathrm{d} x^{\prime} \mathrm{d} y^{\prime}\right] \phi_{m, n}(\lambda) .
\end{aligned}
$$

Since $\phi_{m, n}$ are orthogonal, Eq. (10) must hold independently for each of the $N_{\lambda}$ eigenvectors:

$C_{m, n}^{\mathrm{obs}}(x, y)=\iint C_{m, n}^{\mathrm{sun}}\left(x^{\prime}, y^{\prime}\right) P\left(x-x^{\prime}, y-y^{\prime}\right) \mathrm{d} x^{\prime} \mathrm{d} y^{\prime}$,

which shows that the original problem of convolution or deconvolution of the Stokes vector (Eq. (5)) has been narrowed down to determining the coefficients $C_{m, n}(x, y)$ of the expansion. Since the number of coefficients is equal to the number of observed wavelengths for all four Stokes parameters, $m \times n=4 N_{\lambda}$, applying Eq. (11) or Eq. (5) is, in principle, equivalent and requires the same effort. Interestingly, only a few eigenvectors provide useful information about the Stokes profiles. This implies that we can truncate the expansions in Eqs. (8) and (9) (lower part of these equations) to a much smaller number of coefficients $n=1, \ldots, N_{m}^{\mathrm{pca}} \| \mid N_{\lambda}$. The truncation provides an approximation to the $m$ th component of the Stokes vector $I_{m}^{\text {pca }}(\lambda)$ (hereafter referred to as PCA-reconstructed Stokes profile) that differs by an amount $O_{m}(\lambda)$ from the observed one $I_{m}^{\text {obs }}(\lambda)$ :

$O_{m}(x, y, \lambda)=\left\|I_{m}^{\mathrm{pca}}(x, y \lambda)-I_{m}^{\mathrm{obs}}(x, y, \lambda)\right\|$.

This equation provides a tool to determine where the expansion (Eqs. (8), (9)) must be truncated or, equivalently, a way to determine $N_{m}^{\mathrm{pca}}$. This is done by adding new eigenvectors until the mean (spatial and spectral) difference between the PCAreconstructed and observed Stokes profile is at the level of the
Table 2. Number of PCA coefficients $N_{m}^{\mathrm{pca}}$ needed to reproduce the observed Stokes profiles ( $m=1$ for $I, m=2$ for $Q, m=3$ for $U$, and $m=4$ for $V$ ) at the level of the noise through Eqs. (12) and (13).

\begin{tabular}{c|cccc}
\hline \hline Active region & $N_{1}^{\mathrm{pca}}$ & $N_{2}^{\mathrm{pca}}$ & $N_{3}^{\mathrm{pca}}$ & $N_{4}^{\mathrm{pca}}$ \\
\hline NOAA 12045 & 8 & 10 & 10 & 10 \\
NOAA 12049 & 5 & 5 & 5 & 5 \\
\hline
\end{tabular}

noise in the observations:

$\sigma_{m}^{2} \approx\left(N_{x} N_{y} N_{\lambda}\right)^{-2} \sum_{i, j, k} O_{m}\left(x_{i}, y_{j}, \lambda_{k}\right)^{2}$,

where $N_{x}$ and $N_{y}$ are the total number of spatial points along the $x$ and $y$ directions, respectively. $\sigma_{m}$ refers to the noise in the $m$ th component of the Stokes vector. All these values have been provided in, or can be obtained from, Sect. 2. Table 2 presents the best-fit values of $N_{m}^{\text {pca }}$ obtained through the application of the two above equations. We note that more coefficients are needed to properly reproduce the observed Stokes vector for NOAA 12045 than for NOAA 12049. The reason for this is that NOAA 12049 is very close to disk center $\left(\Theta=6.5^{\circ}\right)$ and therefore there is very little difference between the observed Stokes profiles on the center side and limb side of the penumbra. At larger heliocentric angles (NOAA $12045 ; \Theta=20.5^{\circ}$ ) this is not the case anymore. Moreover, for the latter sunspot the limb-side penumbra displays highly asymmetric three-lobed Stokes profiles similar to those in Borrero et al. $(2004,2005)$. This is undoubtedly a sign of two distinct polarities present in the resolution element, and it explains why more coefficients are needed to reproduce $I^{\text {obs }}$ in NOAA 12045.

The PCA coefficients $C_{m, n}$ for $m=1, \ldots, 4$ and $n=1, \ldots, N_{m}^{\mathrm{pca}}$, that is, obtaining $C_{m, n}^{\text {sun }}$ from $C_{m, n}^{\text {obs }}$ and the known PSF through Eq. (11), were deconvolved by applying ten iterations of a LucyRichardson-like algorithm (Richardson 1972; Lucy 1974) while apodizing the data on the outermost $5 \%$ of the observed field of view for each sunspot. More details about this procedure can be found in Quintero Noda et al. (2015). After the $C_{m, n}^{\text {sun }}$ were obtained, $I_{m}^{\text {sun }}$ can be reconstructed through Eq. (9), but truncating the summation at $N_{m}^{\mathrm{pca}}$ from Table 2 instead of at $N_{\lambda}$. This yields $I^{\text {sun }}(x, y, \lambda)$. As a demonstration of the deconvolution process we compare in Fig. 5 the total circular (top panels) and linear polarization (lower panels), $V_{\text {tot }}$ and $L_{\mathrm{tot}}$, in NOAA 12049 obtained from the originally observed Stokes profiles $\boldsymbol{I}^{\mathrm{obs}}$ (left) and the deconvolved Stokes profiles $\boldsymbol{I}^{\text {sun }}$ using a truncated PCA expansion $\left(\simeq\right.$ symbols in Eqs. (8) and (9)). $V_{\text {tot }}$ and $L_{\text {tot }}$ are obtained as the wavelength integral of $\|V(\lambda)\|$ and $\left.\sqrt{\{} Q^{2}(\lambda)+U^{2}(\lambda)\right\}$, respectively. At high frequencies (narrow-angle) data are irredeemably lost and the deconvolution process cannot recover them. This is not the case at low frequencies (wide-angle scattered light), where the information can be efficiently recovered. Therefore, we can consider that after the deconvolution, the spatial resolution is given by the width of the narrow-angle Gaussian: $\sigma_{n}=0.18^{\prime \prime}$ (Sect. 4.1.2). This value is also supported by the power spectra of the granulation (see Sect. 2).

\subsection{Inversion of Stokes profiles}

We now apply the SIR inversion code (Stokes inversion based on response functions; Ruiz Cobo \& del Toro Iniesta 1992) to $\boldsymbol{I}^{\text {sun }}(\lambda)$ to infer the kinematic, thermodynamics, and magnetic properties of the solar atmosphere in the region within 

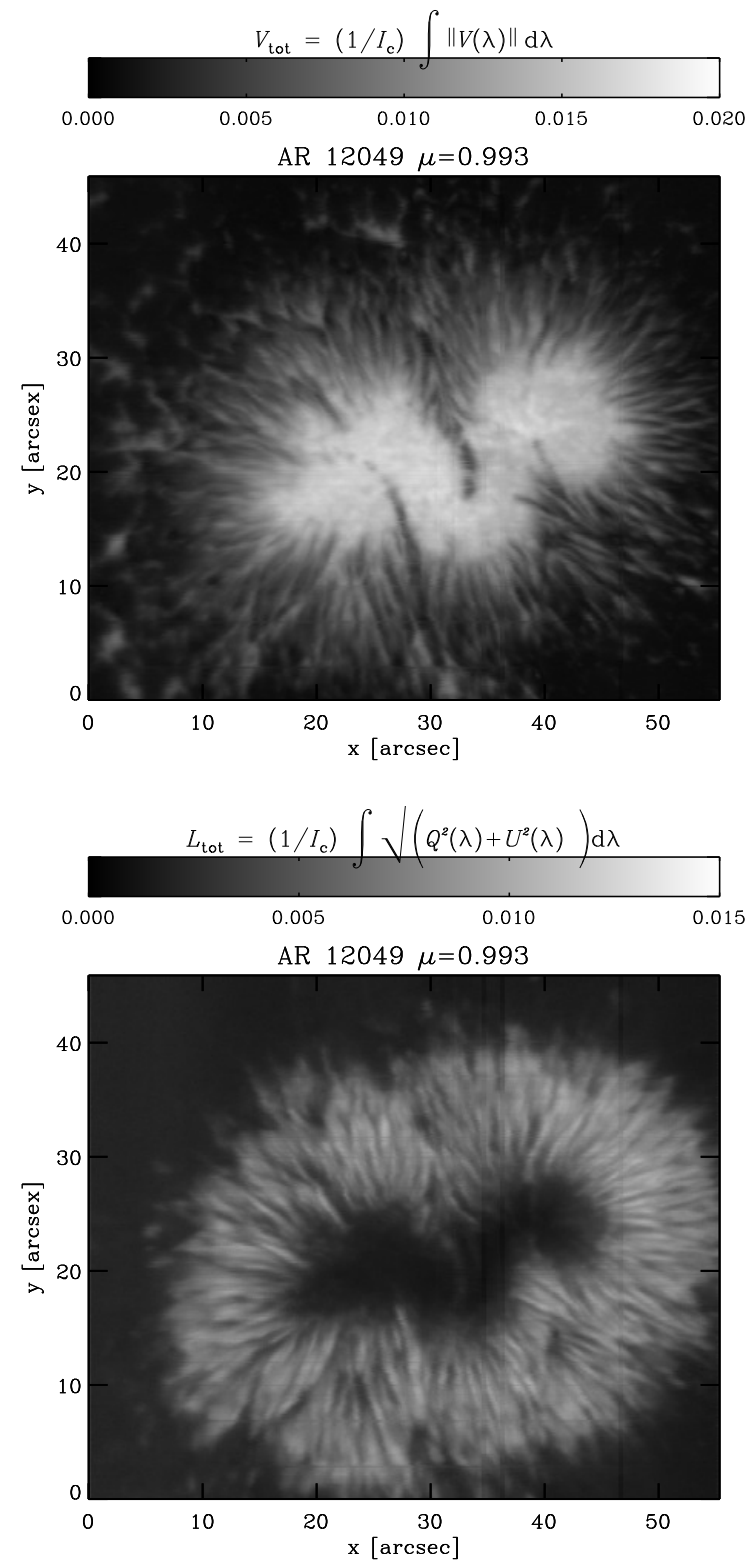
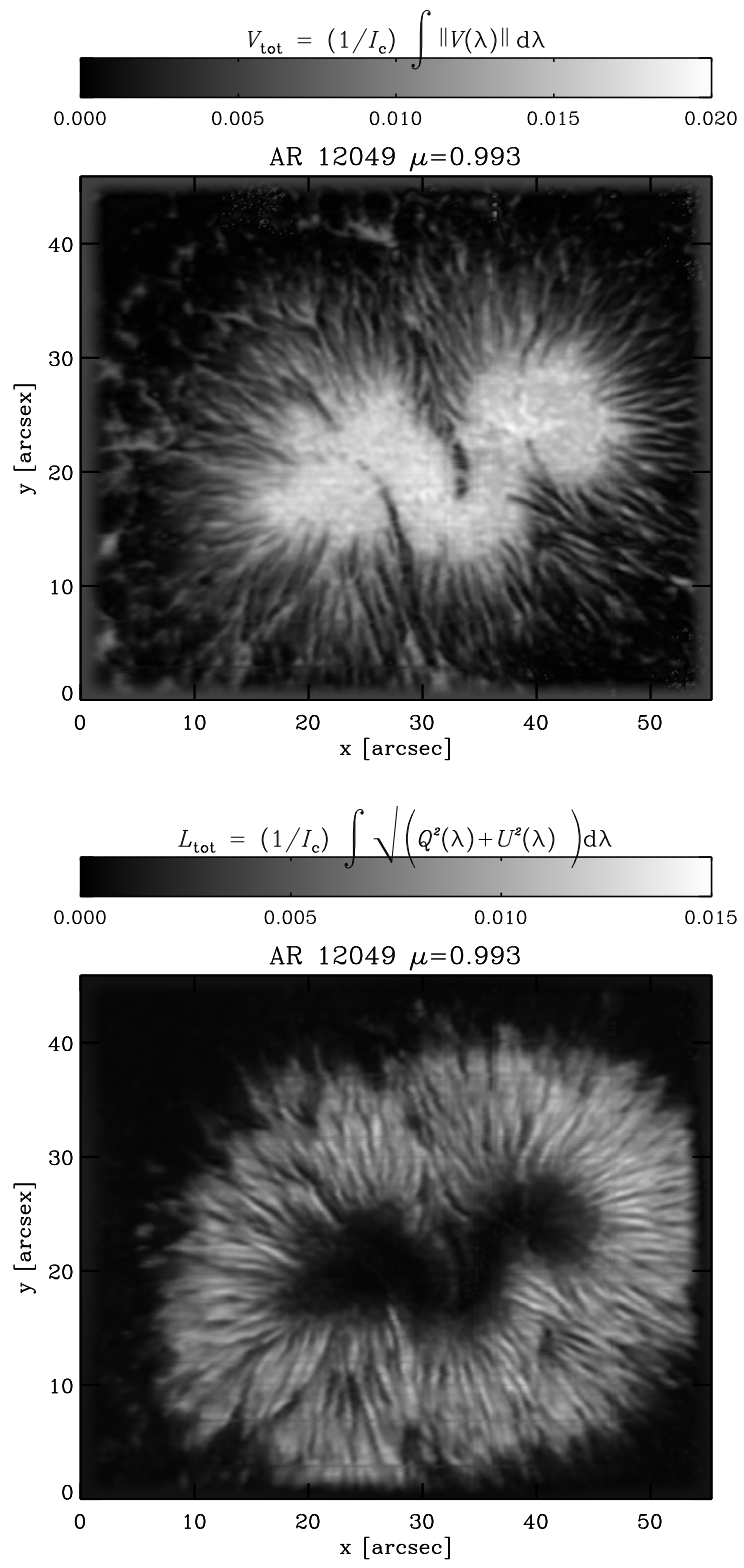

Fig. 5. Comparison between the total circular (top) and linear (bottom) polarization using the original observed Stokes vector $\boldsymbol{I}^{\text {obs }}($ left) and the deconvolved Stokes vector $\boldsymbol{I}^{\text {sun }}$ (right) after truncating the PCA expansion. The frame around the deconvolved maps (better seen in the upper right panel) appears as a consequence of apodization before deconvolution.

the red rectangles in Fig. 1. The inversion code employs an initial guess model for the solar atmosphere as a function of the continuum optical depth at a reference wavelength of $500 \mathrm{~nm}$, $\log \tau_{5}$, referred to as $\boldsymbol{M}_{0}\left(\log \tau_{5}\right)$, to solve the radiative transfer equation and obtain a synthetic Stokes vector $\boldsymbol{I}^{\mathrm{syn}}(\lambda)$. This synthetic Stokes vector is then compared with the real one $\boldsymbol{I}^{\text {sun }}(\lambda)$ through a $\chi^{2}$ merit function. The initial model is then perturbed, $\boldsymbol{M}_{1}=\boldsymbol{M}_{0}+\delta \boldsymbol{M}$, so as to minimize the $\chi^{2}$ merit function. To that end, the perturbation $\delta \boldsymbol{M}$ is obtained through a LevenbergMarquardt algorithm (Press et al. 1986) and singular value decomposition method (Golub \& Kahan 1965). The perturbations are introduced at specific $\log \tau_{5}$ positions called nodes, with the final optical-depth dependence $\delta \boldsymbol{M}\left(\log \tau_{5}\right)$ being obtained by interpolating between the nodes. The perturbative process is repeated until a minimum in $\chi^{2}$ is reached. The resulting atmospheric model could be taken as representative of the physical conditions of the solar plasma, but as a result of the dependence on the results from the initial guess model, we have repeated the inversion procedure ten different times employing random initial models $\boldsymbol{M}_{0}$. The model that yielded the best $\chi^{2}$ was adopted as the final solution.

The process described above must be applied independently to each $(x, y)$ pixel contained in the red rectangles in Fig. 1. We then obtain $\boldsymbol{M}\left(x, y, \log \tau_{5}\right)$, which consists of the threedimensional structure of the line-of-sight-velocity $v_{\text {los }}$, temperature $T$, magnetic field strength $B$, inclination of the magnetic field with respect to the observer line of sight $\gamma$, and finally the azimuth of the magnetic field on the plane perpendicular to the observer line of sight $\psi$.

Our inversions were carried out with three nodes in $T$ and two nodes in $v_{\mathrm{los}}, B, \gamma$, and $\psi$, respectively. This adds up to a total of ten free parameters that were used to fit at each spatial pixel $(x, y)$, the solar Stokes vector $\boldsymbol{I}^{\text {sun }}(\lambda)$ containing $4 N_{\lambda}=$ 2400 data points. The node selection remains the same until Sect. 5.4, where a more complex situation is considered. When constructing the $\chi^{2}$ merit function that measures the difference 


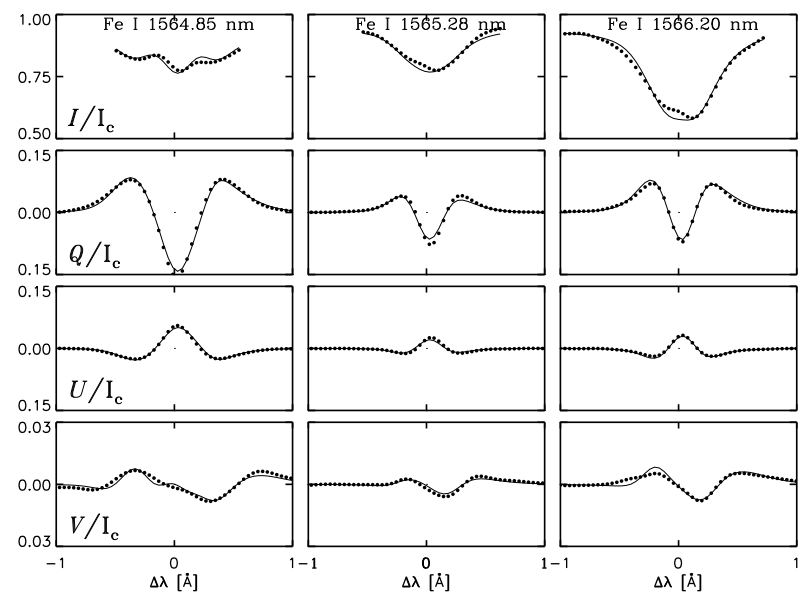

Fig. 6. Example of the observed (after PCA deconvolution; filled circles) and best-fit (solid lines) Stokes profiles resulting from the inversion (see Sect. 4.3) in a intraspinal pixel. From top to bottom: results for $I, Q, U$, and V. From left to right: results for Fe I $1565.85 \mathrm{~nm}$, $\mathrm{Fe}$ I $1565.28 \mathrm{~nm}$, and Fe I $1566.20 \mathrm{~nm}$ (see Table 1). The location of this particular pixel is indicated in Fig. 8 with a black square.

between the synthetic $\boldsymbol{I}^{\text {syn }}(\lambda)$ and solar $\boldsymbol{I}^{\text {sun }}(\lambda)$ Stokes vector, the polarization profiles $Q, U$ and $V$ were given twice the statistical weight of $I$. This was done because Stokes $I$ is more affected by unidentified molecular blends and leftover fringes that are not fully corrected during data reduction (see Sect. 2). We note that at each iteration step and before being compared with $\boldsymbol{I}^{\text {sun }}$, the synthetic Stokes vector $I^{\text {syn }}$ was convolved with a Gaussian profile with $\sigma=70 \mathrm{~m} \AA$. This was done to include the effects of the spectrograph profile $g(\lambda, \sigma)$ that remained unaccounted for after the removal of the spectral veil (see Sect. 3). We emphasize that we employed only one component during the inversion. No nonmagnetic component was used to model the stray light because this was accounted for in the deconvolution (Sect. 4.2).

Figure 6 shows an example of the observed after PCA deconvolution (filled circles) and best-fit (solid lines) Stokes profiles in the three observed spectral lines. This is the result for a pixel that corresponds to a penumbral intraspine in NOAA 12049 (black square in Fig. 8). Because less weight was given to the intensity during the inversion (because of blends and leftover fringes), the fits in Stokes $I$ are always of lower quality than in $Q, U, V$. Since the selected pixel is located in an intraspine, Stokes $V$ features several lobes. Although it uses only linear gradients (i.e., two nodes in $v_{\text {los }}, B, \gamma$, and $\psi$ ), the inversion works well in fitting these multilobed circular polarization signals. Outside the intraspines, Stokes $V$ regains it regular shape, and the quality of the fits improves significantly.

The three-dimensional distribution of the kinematic and thermodynamics parameters provided by the inversion will be discussed in a future paper. In this work we focus only on the magnetic field. The radiative transfer equation allows us to determine the magnetic field in spherical coordinates in the observer's reference frame: $\boldsymbol{B}=(B, \gamma, \psi)$. Instead, it is more useful to analyze the magnetic field in cylindrical coordinates in the local reference frame: $\boldsymbol{B}=\left(B_{r}, B_{\theta}, B_{z}\right)$, where $B_{z}$ corresponds to the direction perpendicular to the solar surface, and $B_{r}$ and $B_{\theta}$ are the magnetic field components in polar coordinates on the plane parallel to the solar surface with origin at the sunspot centers. To this end, we have employed the methods described by Borrero et al. (2008) and Borrero \& Ichimoto (2011) to convert from the observer's to the local reference frame. This method solves the $180^{\circ}$ ambiguity in the azimuth of the magnetic field vector by choosing at each pixel $(x, y)$ on the solar surface either $\psi$ or $\psi+\pi$ so that the magnetic field becomes as radial as possible. This yields $B_{r}\left(x, y, \log \tau_{5}\right), B_{\theta}\left(x, y, \log \tau_{5}\right)$, and $B_{z}\left(x, y, \log \tau_{5}\right)$.

\section{Results and discussion}

\subsection{Field-free gaps and return flux}

Figures 7 and 8 show the maps at an optical depth of $\log \tau_{5}=0$ of the magnetic field strength $B$ (left) and the vertical component of the magnetic field $B_{z}$ in the local reference frame (right) in the regions denoted by the red rectangles in Fig. 1 for NOAA 12045 and 12049 , respectively. In these plots the black arrows denote the projection of the magnetic field $\boldsymbol{B}$ onto the solar surface $\left(B_{r}, B_{\theta}\right)$. The length of each arrow is proportional to $\left.\sqrt{\{} B_{r}^{2}+B_{\theta}^{2}\right\}$. For better visualization we show the vectors only every other pixel in the vertical and horizontal directions. White contours on the $B$ maps (left) enclose those regions where $B \leq 500$ Gauss, while on the $B_{z}$ maps (right) they enclose regions where $B_{z} \leq 0$ (i.e., magnetic field lines returning to the solar surface).

The most noticeable pattern in these figures is the very wellknown spine and intraspine penumbral structure (Lites et al. 1993) described in Sect. 1: regions of strong and vertical magnetic fields (spines) interlaced with weaker and more horizontal magnetic fields (intraspines).

The regions where the magnetic field strength is lower than 500 Gauss represent only $0.5 \%$ and $0.2 \%$ of the total area in Figs. 7 (left) and 8 (left), respectively. These areas would be even smaller if we had employed the 300 Gauss limit found by Spruit et al. (2010). At close inspection we note that these tiny regions where the magnetic field is below 500 Gauss correspond to pixels in the outer penumbra where granules enter the sunspot. A clear example of this can be found in the white contours in the left panel of Fig. $8(B \leq 500 \mathrm{G})$ at position $\left(x \approx 8.4^{\prime \prime}\right.$, $\left.y \approx 3.7^{\prime \prime}\right)$. This region corresponds to a bright granule in the outer penumbra inside the red rectangle in the bottom panel of Fig. 1 at position $\left(x \approx 53^{\prime \prime}, y \approx 17^{\prime \prime}\right)$. These results rule out the existence, at an optical depth of $\log \tau_{5}=0$, of dynamically weak magnetic fields (cf. Scharmer 2008; Spruit et al. 2010), and even more strongly so the presence of field-free gaps in the deep photospheric layers of the penumbra (Spruit \& Scharmer 2006; Scharmer \& Spruit 2006). This agrees with previous results obtained from Hinode/SP data (Borrero \& Solanki 2008; Puschmann et al. 2010; Ruiz Cobo \& Asensio Ramos 2013; Tiwari et al. 2013, 2015) and SST/CRISP data (Scharmer et al. 2008, 2013; Scharmer \& Henriques 2012). However, it must be borne in mind that the information at $\log \tau_{5}=0$ provided by the spectral lines employed in this work (Sect. 2; see also Table 1) is much more reliable than the information at the same optical depth provided by the spectral lines (Fe I line pair at $630 \mathrm{~nm}$ ) employed in previous investigations (see Sect. 5.2). Finally, it is worth mentioning that the results for NOAA 12049 are of particular importance because this sunspot is located very close to disk center, thereby allowing us to probe slightly deeper photospheric layers than NOAA 12045.

Field-free gaps or dynamically weak magnetic fields in the penumbra have previously been ruled out (Mathew et al. 2003; Borrero et al. 2004, 2005; Bellot Rubio et al. 2004; Cabrera Solana et al. 2008) from observations of the same deep-forming $\mathrm{Fe}$ I spectral lines around $1565 \mathrm{~nm}$ as we used here (see Table 1). However, these older investigations were carried out with data at relatively low spatial resolution $(1 \mathrm{arcsec})$ and did not account for wide-angle scattered light. 

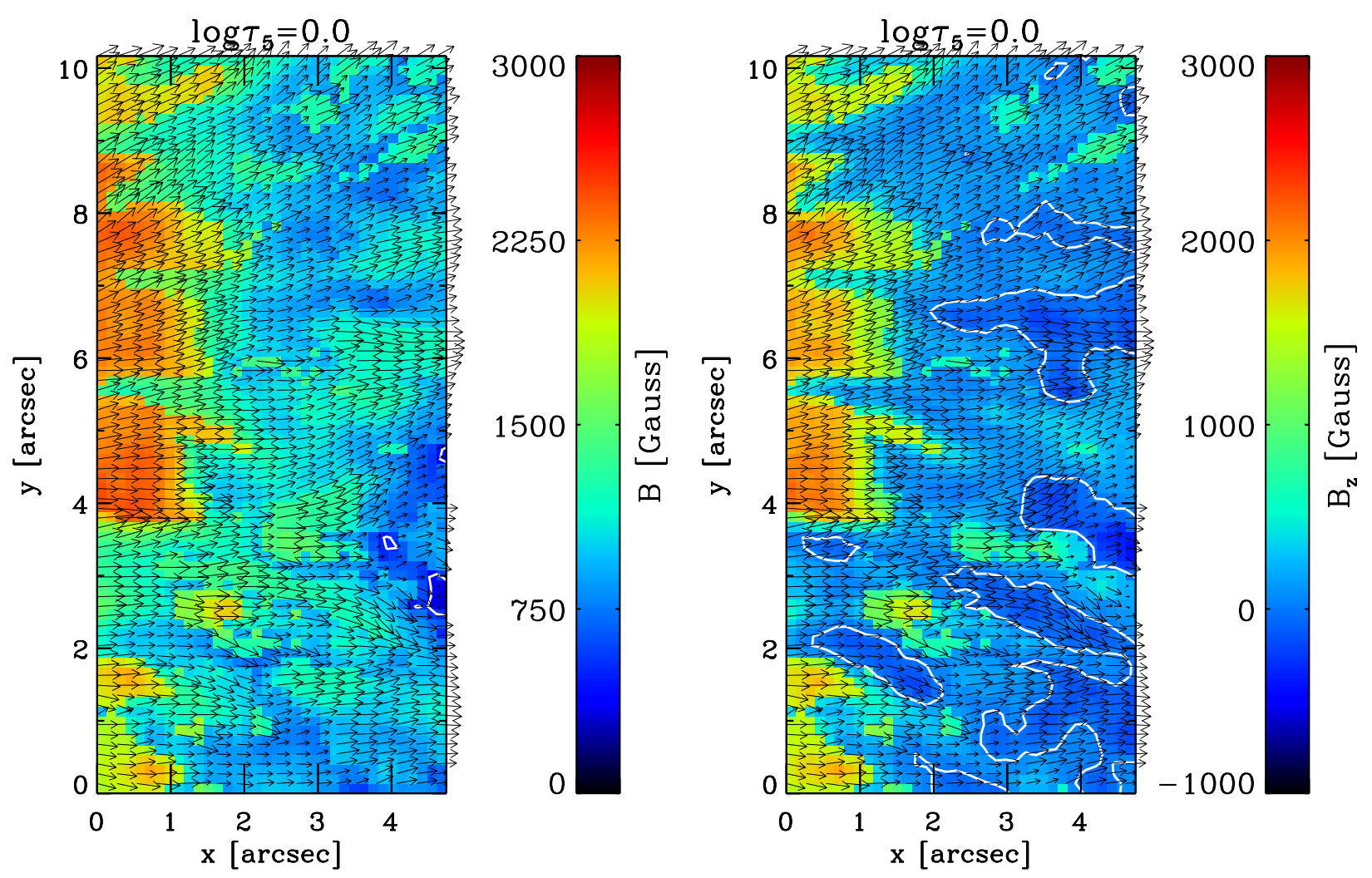

Fig. 7. Magnetic field strength $B\left(x, y, \log \tau_{5}=0\right)($ left $)$ and vertical component of the magnetic field in the local reference frame $B_{z}\left(x, y, \log \tau_{5}=0\right)$ (right) in NOAA 12045 observed on April 24, 2014 at $\Theta=20.5^{\circ}$. White contours in the left and right panels indicate regions where $B<500$ Gauss and $B_{z}<0$, respectively. Black arrows indicate the projection of the magnetic field vector $\boldsymbol{B}$ on the plane of the solar surface. This region corresponds to the red rectangle in Fig. 1 (top panel). This map was obtained from inverting the deconvolved data assuming $p_{w}=0.2(20 \%$ wide-angle scattered light).

Regions where $B_{z}<0$ represent $19.6 \%$ and $3.0 \%$ of the total area in the right panels of Figs. 7 and 8, respectively. The much larger region of magnetic flux return in NOAA 12045 is probably due to the presence of a nearby plage with opposite -polarity magnetic fields outside the sunspot. This also shortens the radial extension of the penumbra on the limb side of NOAA 12045 as compared to 12049 . These numbers do not fully agree with the values given by Ruiz Cobo \& Asensio Ramos (2013), who found that at $\log \tau_{5}=0$, about $28 \%$ of the penumbra harbors magnetic field lines returning into the solar surface. An additional difference between our result and those from Ruiz Cobo \& Asensio Ramos (2013) and Scharmer et al. (2013) is that the regions of return flux detected here also enclose the central core of the intraspines, not only their lateral boundaries (see left panels in Figs. 7 and 8).

The discrepancy in the results might have several sources. On the one hand, as has been mentioned by Ruiz Cobo \& Asensio Ramos (2013, see also Sect. 5.2), $B_{z}$ at $\log \tau_{5}=0$ is not very well constrained by spectropolarimetric observations of the $\mathrm{Fe}$ I spectral lines at $630 \mathrm{~nm}$ (Hinode/SP and SST/CRISP). From this point of view, our results at this optical depth are to be preferred. On the other hand, as we show in Sect. 5.3, our results on $B_{z}$ are heavily dependent on the amount (Eq. (6); Sect. 4.1) of scattered light, $p_{w}$, employed in the PSF. Since our knowledge of GRIS/GREGOR PSF is very limited (Sect. 3) compared to Hinode/SP (Suematsu et al. 2008; Danilovic et al. 2008; van Noort 2012), the $28 \%$ of return-flux area provided by Ruiz Cobo \& Asensio Ramos (2013) might be considered more reliable. Given that both sets of data have their shortcomings, the total amount of return flux present in the penumbra and its spatial distribution appears to be still open to debate. More investigations need to be carried out on this subject before a more comprehensive answer can be given.

\subsection{How deep do we probe?}

Throughout this paper we have stated several times that the Fe I spectral lines at $1565 \mathrm{~nm}$ employed in this work provide more reliable information about the deep photospheric layers than the commonly used Fe I spectral lines at $630 \mathrm{~nm}$. In Sect. 2 we ascribed this to the lower $\mathrm{H}^{-}$opacity at $1565 \mathrm{~nm}$ than at $630 \mathrm{~nm}$, plus the higher excitation potential of the former spectral lines compared to the latter. However, this is only a qualitative explanation. In this section we provide a more quantitative explanation.

To that end we determined the depth of the optical depth unity level as a function of wavelength $z\left(\tau_{\lambda}=1\right)$ for different atmospheric models: the granular model from Borrero \& Bellot Rubio (2002), hot and cool umbral models from Collados et al. (1994), and finally the spatially averaged penumbral model obtained from the inversions of NOAA 12049 described in Sect. 4.3. The results are shown in Fig. 9. All curves in this figure have been shifted vertically so that $z\left(\tau_{\lambda}=1\right)=0$ for a wavelength of $500 \mathrm{~nm}$. As expected, all curves follow the opacity as a result of the bound-bound and bound-free transitions of the $\mathrm{H}^{-}$ion (Chandrasekhar \& Breen 1946), but each is modulated by the density and temperature of the different models. The height difference between the continuum level $\tau_{\lambda}=1$ or $\log \tau_{\lambda}=0$ at $630 \mathrm{~nm}$ and $1565 \mathrm{~nm}$ is $-36 \mathrm{~km}$ (granular model; red curve), $-62 \mathrm{~km}$ (cool-large umbra; green curve) and $-71 \mathrm{~km}$ 

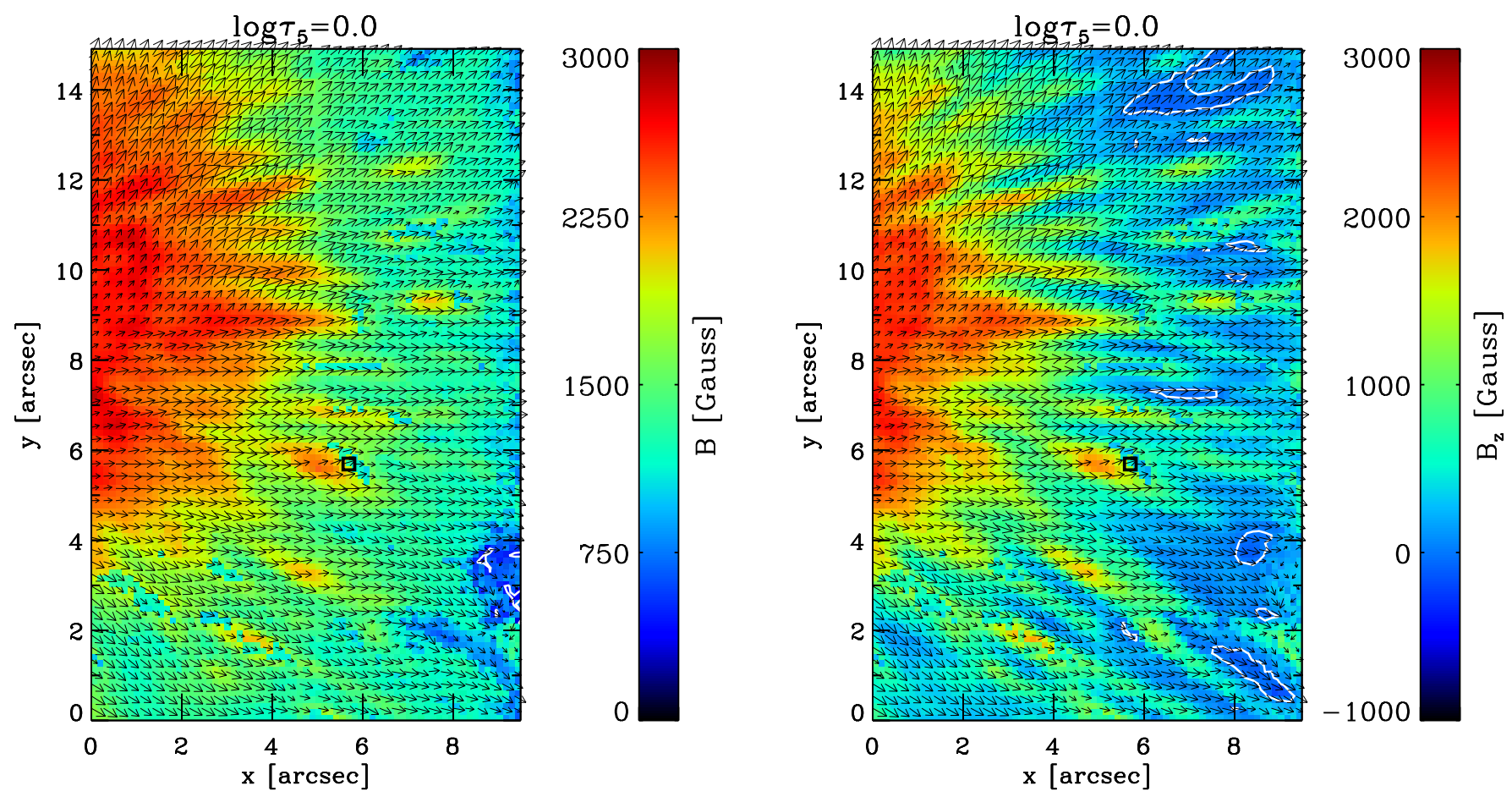

Fig. 8. Magnetic field strength $B\left(x, y, \log \tau_{5}=0\right)($ left $)$ and vertical component of the magnetic field in the local reference frame $B_{z}\left(x, y, \log \tau_{5}=0\right)$ (right) in NOAA 12049 observed on May 3, 2014 at $\Theta=6.5^{\circ}$. White contours in the left and right panels indicate regions where $B<500$ Gauss and $B_{z}<0$, respectively. Black arrows indicate the projection of the magnetic field vector $\boldsymbol{B}$ onto the plane of the solar surface. This region corresponds to the red rectangle in Fig. 1 (bottom panel). This map was obtained from inverting the deconvolved data assuming $p_{w}=0.2(20 \%$ wide-angle scattered light).

(hot-small umbra; blue curve), and $-75 \mathrm{~km}$ (average penumbra; black curve), meaning that the continuum level is formed some $60-70 \mathrm{~km}$ deeper (i.e., half a pressure scale height) at $1565 \mathrm{~nm}$ than at $630 \mathrm{~nm}$ in sunspots but only about $30 \mathrm{~km}$ deeper in the quiet-Sun.

However, the formation height of the continuum level tells only part of the story. We must also consider the formation of the spectral lines themselves, which depends on their excitation potential, oscillator strength, and electronic configuration. This can be achieved by means of the so-called response functions $\mathcal{R}\left(\log \tau_{5}, \lambda\right)$ (del Toro Iniesta 2003). We calculated the response functions of the spectral lines at $1565 \mathrm{~nm}$ (Table 1) and at $630 \mathrm{~nm}$ using the average penumbral model mentioned previously. The contribution from each of the four Stokes parameters has been taken into account following the method described in Borrero et al. (2014). Figure 10 presents the wavelength-integrated response functions to the magnetic field strength $B$ (top-left), magnetic field inclination with respect to the observer line of sight $\gamma$ (top-right), temperature $T$ (bottom left), and line-of-sight velocity $v_{\text {los }}$ (bottom right). These figures demonstrate that the nearinfrared (NIR) spectral lines at 1565 observed by GRIS at the GREGOR telescope and employed in this work are much more sensitive to the magnetic field $B$ at $\log \tau_{5}=0$ in their peaksensitivity (red curves in the top left panel) than the visible spectral lines at $630 \mathrm{~nm}$ observed by Hinode/SP and the CRISP instrument at the SST telescope (blue curves in the top left panel). In particular, the response function to $B$ in the infrared lines at $\log \tau_{5}=0$ is about $70 \%$ of its highest value, while this number decreases to $25 \%$ for the spectral lines in the visible. Moreover, the information on the magnetic field conveyed by the $630 \mathrm{~nm}$ lines is spread over a much wider range of optical depths, making it more difficult to isolate the information at $\log \tau_{5}=0$ than for the NIR lines, whose contribution comes from a much narrower range. This suggests that the results obtained at $\log \tau_{5}=0$ from inverting the $\mathrm{Fe}$ I lines at $630 \mathrm{~nm}$, in particular inversions carried out with two nodes (Scharmer et al. 2013), tend to be an extrapolation toward deeper layers ${ }^{1}$ from the results between $\log \tau_{5} \in[-2,-1]$. In addition, the peak contribution is much closer to $\log \tau_{5}=0$ in the NIR spectral lines than in the visible, with the center-of-gravity of $\mathcal{R}$ being located at $\log \tau_{5} \approx-0.6$ and at $\log \tau_{5} \approx-1.3$, respectively. This implies that the information about the magnetic field strength $B$ comes from an optical depth of about $10^{-0.6} / 10^{-1.3} \approx 5$ times larger in the NIR spectral lines employed in this work.

\subsection{Dependence on the PSF used to deconvolve}

In Sect. 4.1 we obtained a simple empirical PSF, $P(x, y)$, for the instrument GRIS attached to the GREGOR telescope. The PSF was modeled through a narrow- and a wide-angle Gaussian profile characterized by $\sigma_{n}, p_{n}$ and $\sigma_{w}, p_{w}$ parameters, respectively (Eq. (6)). The latter two parameters were rather uncertain, with possible values such as $\sigma_{w} \in\left[10^{\prime \prime}, 30^{\prime \prime}\right]$ and $p_{w} \in[0.2,0.3]$. The results presented in Sect. 5.1 (see also Figs. 7 and 8) were obtained with $p_{w}=0.2, \sigma_{w}=20^{\prime \prime}$. Motivated by Schlichenmaier \& Franz (2013) we want to investigate whether our results depend on the amount of scattered light $p_{w}$. We have therefore repeated the PCA deconvolution (Sect. 4.2) and Stokes inversion (as described in Sect. 4.3) using different PSF parameters. As a first experiment we have simply inverted veil-corrected but

1 This is the case of inversions carried out with SIR (Ruiz Cobo \& del Toro Iniesta 1992) or NICOLE (Socas-Navarro et al. 2015), but not SPINOR (Frutiger et al. 1999) because the first two codes spread the number of nodes evenly in $\log \tau_{5}$ with the first two nodes being located at the top and bottom of the atmosphere. The latter, however, allows the user to choose their locations. 


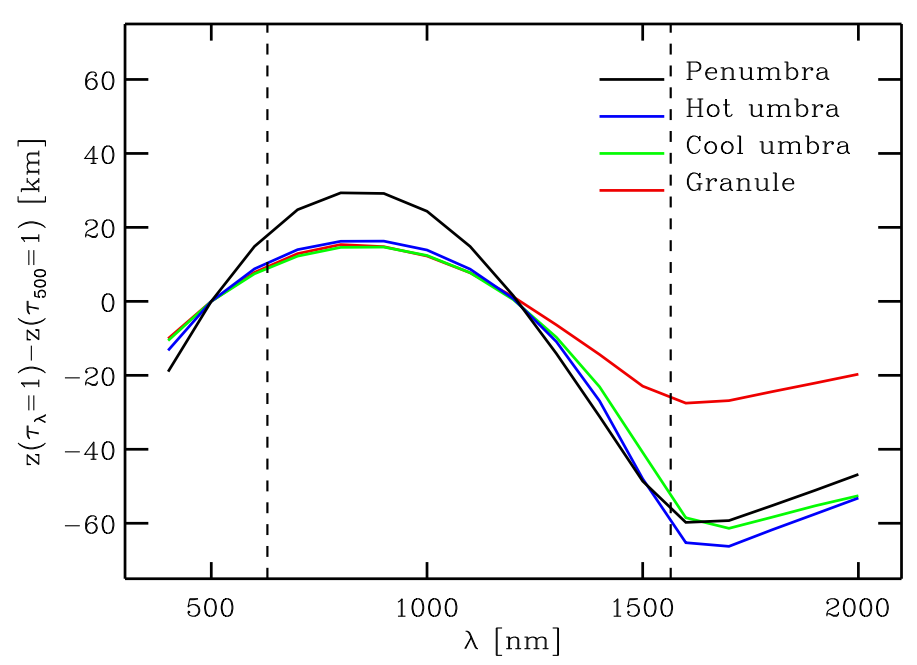

Fig. 9. Height at which the continuum level $z\left(\tau_{\lambda}=1\right)$ is formed, with respect to the height at a wavelength of $500 \mathrm{~nm}$ as a function of wavelength. The color curves indicate the different models: red (granular model), blue (hot-small umbral model), green (cool-large umbral model), and black (penumbral model). The two vertical dashed lines are located at $\lambda=630 \mathrm{~nm}$ and $\lambda=1565 \mathrm{~nm}$.

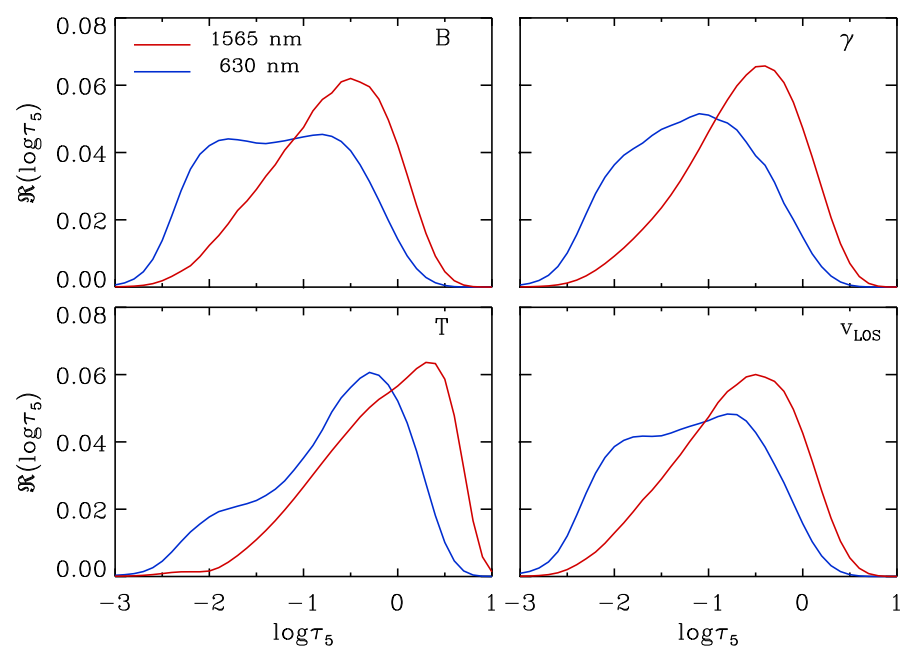

Fig. 10. Wavelength-integrated response function $\mathcal{R}$ as a function of the logarithm of the optical depth at a reference wavelength of $500 \mathrm{~nm}$, $\log \tau_{5}$, to the magnetic field strength $B$ (top left), magnetic field inclination with respect to the observer line of sight $\gamma$ (top right), temperature $T$ (lower left), and line-of-sight velocity $v_{\text {los }}$ (lower right). Red curves correspond to the integration over the observed spectral lines in this work (see Table 1), while the blue lines correspond to a wavelength integration over the Fe I line pair at $630 \mathrm{~nm}$. The atmospheric model employed to calculate the response functions is the spatially averaged penumbral model obtained from the inversions (see Sect. 4.3).

undeconvolved raw data, that is, skipping Sect. 4.2 in the analysis. Next, we deconvolved before inversion with the following combinations: (a) $p_{w}=0$; (b) $p_{w}=0.2, \sigma=20^{\prime \prime}$; and (c) $p_{w}=0.4, \sigma=10^{\prime \prime}$. The other two parameters were always kept to $p_{n}=1-p_{w}$ and $\sigma_{n}=0.18^{\prime \prime}$. Table 3 summarizes our findings for each experiment in terms of the percentage of the total area covered by regions with $B<500$ Gauss and $B_{z}<0$ at $\log \tau_{5}=0$. Results for case (b) have been presented in Sect. 5.1 (see also Figs. 7 and 8).

Clearly, the penumbral area featuring regions where the magnetic field returns to the solar surface $\left(B_{z}<0\right)$ strongly depends
Table 3. Percentage of the analyzed penumbral area that harbors weak fields $(B<500$ Gauss) or magnetic field lines returning into the solar surface $\left(B_{z}<0\right)$ for different PSF parameters.

\begin{tabular}{l|cc|cc}
\hline \hline \multirow{2}{*}{ Dataset } & \multicolumn{2}{|c|}{ NOAA } & 12045 & \multicolumn{2}{c}{ NOAA 12049} \\
& $B<500$ & $B_{z}<0$ & $B<500$ & $B_{z}<0$ \\
\hline \multirow{2}{*}{ raw } & $0.1 \%$ & $6.1 \%$ & $0.0 \%$ & $0.5 \%$ \\
$p_{w}=0$ & $0.8 \%$ & $11.9 \%$ & $0.2 \%$ & $0.3 \%$ \\
$p_{w}=0.2, \sigma_{w}=20^{\prime \prime}$ & $0.5 \%$ & $19.6 \%$ & $0.2 \%$ & $3.0 \%$ \\
$p_{w}=0.4, \sigma_{w}=10^{\prime \prime}$ & $0.1 \%$ & $31.6 \%$ & $0.3 \%$ & $12.7 \%$ \\
\hline
\end{tabular}

Table 4. Same as Table 3, but employing three nodes for $v_{\mathrm{los}}, B, \gamma$, and $\phi$ instead of two nodes.

\begin{tabular}{l|cc|cc}
\hline \hline \multirow{2}{*}{ Dataset } & \multicolumn{2}{|c|}{ NOAA } & 12045 & \multicolumn{2}{c}{ NOAA } & 12049 \\
& $B<500$ & $B_{z}<0$ & $B<500$ & $B_{z}<0$ \\
\hline raw & $0.0 \%$ & $6.0 \%$ & $0.0 \%$ & $0.5 \%$ \\
$p_{w}=0$ & $0.3 \%$ & $12.6 \%$ & $0.5 \%$ & $0.0 \%$ \\
$p_{w}=0.2, \sigma_{w}=20^{\prime \prime}$ & $0.3 \%$ & $27.8 \%$ & $0.1 \%$ & $2.3 \%$ \\
$p_{w}=0.4, \sigma_{w}=10^{\prime \prime}$ & $0.1 \%$ & $48.4 \%$ & $0.2 \%$ & $10.2 \%$ \\
\hline
\end{tabular}

on the amount of scattered light $p_{w}$ employed to model the PSF. As $p_{w}$ increases, the areas harboring return flux becomes larger. The area covered by weak magnetic fields $(B<500)$ is independent of $p_{w}$, however.

\subsection{Dependence on the number of nodes employed in the inversion}

So far, the results we presented were obtained by employing, during the inversion process, three nodes in the temperature $T$, and two nodes in the line-of-sight velocity $v_{\text {los }}$, magnetic field strength $B$, inclination of the magnetic field with respect to the observer line of sight $\gamma$, and angle of the magnetic field in the plane perpendicular to the observer line of sight $\psi$ (see Sect. 4.3). Using two nodes in these physical parameters assumes that each of them varies linearly with the logarithm of the optical depth $a+b * \log \tau_{5}$, with the slope $b$ and zero crossing $a$ being different for $B, \gamma$, etc. Depending on the sign of the slope, this implies that a given physical parameter can either increase or decrease with height in the atmosphere. A slightly more realistic, albeit complex, situation would be to allow for three nodes instead of two (i.e., quadratic dependence of the physical parameters on $\log \tau_{5}$ ) and hence allowing them to first increase with height and then decrease, or vice versa. To determine how our results depend on the choice of nodes, we repeated all inversions in Sect. 5.3, but employed three nodes in $v_{\text {los }}, B, \gamma$, and $\phi$. Table 4 shows the percentage of the penumbral area harboring weak fields $(B<500$ Gauss $)$ and field lines returning to the solar surface $\left(B_{z}<0\right)$ employing three nodes. From comparing Table 4 with Table 3, we conclude that the size of flux-return areas significantly depends on whether each physical parameter is modeled with two or three nodes. The area of weak fields is the same in both cases, however.

\section{Conclusions}

We have studied the magnetic field topology in the penumbra of two sunspots at the deepest layers of the solar photosphere. This was done through inverting the radiative transfer equation applied to spectropolarimetric data (i.e., full Stokes vector I) 
of three Fe I spectral lines at $1565 \mathrm{~nm}$ to retrieve the magnetic field $\boldsymbol{B}$.

The estimated spatial resolution of the data we employed is $0.4-0.45^{\prime \prime}$ and the noise level is $10^{-3}$. Moreover, the observed spectral lines are better suited to study the magnetic field in the deep photosphere than the widely used Fe I spectral lines at $630 \mathrm{~nm}$ because the lines at $1565 \mathrm{~nm}$ convey information from deeper photospheric layers, and the Zeeman splitting is about three times larger than in the lines at $630 \mathrm{~nm}$.

To account for the degradation of the data by straylight (i.e., wide-angle scattered light) within the instrument, we applied before the inversion a PCA deconvolution method using an empirical PSF. Our results show no evidence of weakfield regions $(B<500)$, let alone of dynamically weak fields (Spruit et al. 2010) or field-free regions (Scharmer \& Spruit 2006; Spruit \& Scharmer 2006) in the deepest regions of the photosphere $\left(\log \tau_{5}=0\right)$. This agrees with previous observational results, in particular with Borrero \& Solanki (2008, 2010), Tiwari et al. (2013) and with three-dimensional MHD simulations of sunspot fine-structure (Rempel et al. 2009; Nordlund \& Scharmer 2010; Rempel 2011, 2012). These results are independent of the amount of stray light used in the PSF and independent of the inversion setup (i.e., number of nodes). No previous work has ruled out field-free plasma deep beneath the sunspot. It is perfectly plausible that at some point underneath the sunspot (i.e., below the magnetopause; Jahn \& Schmidt 1994) normal field-free convection resumes. The question is wether this occurs sufficiently close to $\log \tau_{5}=0$ so as to explain the penumbral brightness. This is precisely what we rule out here.

On the other hand, the amount of flux returning back into the solar surface $\left(B_{z}<0\right)$ within the penumbra is highly dependent on the amount of stray light considered and, consequently, we refrain from drawing conclusions at this point.

In summary, we have addressed all major concerns raised by Spruit et al. (2010), Scharmer \& Henriques (2012), and Scharmer et al. (2013): we used high-spatial resolution observations (indeed the highest ever at $1565 \mathrm{~nm}$ ) of spectropolarimetric data (Scharmer \& Henriques 2012) that conveys very reliable information about the magnetic field in the deep photosphere (Spruit et al. 2010). We also deconvoled the data with several empirically determined PSFs, which allowed us to remove the need for the so-called non-magnetic filling factor (Scharmer et al. 2013). In all cases, no traces of regions with $B \leq 500$ Gauss have been found at $\log \tau_{5}=0$.

Acknowledgements. The 1.5-m GREGOR solar telescope was built by a German consortium under the leadership of the Kiepenheuer-Institut für Sonnenphysik in Freiburg with the Leibniz-Institut für Astrophysik Potsdam, the Institut für Astrophysik Göttingen, and the Max-Planck-Institut für Sonnensystemforschung in Göttingen as partners, and with contributions by the Instituto de Astrofísica de Canarias and the Astronomical Institute of the Academy of Sciences of the Czech Republic. We are very grateful to the engineering, operating and technical staff at the GREGOR Telescope: Andreas Fischer, Olivier Grassin, Roberto Simoes, Clements Halbgewachs, Thomas Kentischer, Thomas Sonner, Peter Caligari, Michael Wiessschädel, Frank Heidecke, Stefan Semeraro, and Oliver Wiloth. We are also indebted to Franz Kneer and an anonymous referee for their comments on the paper. A.A.R. acknowledges financial support by the Spanish Ministry of Economy and Competitiveness through project AYA2014-60476-P and the Ramón y Cajal fellowships. This research has made use of NASA's Astrophysics Data System.

\section{References}

Allende Prieto, C., Asplund, M., \& Fabiani Bendicho, P. 2004, A\&A, 423, 1109 Anstee, S. D., \& O'Mara, B. J. 1995, MNRAS, 276, 859 Asensio Ramos, A., \& López Ariste, A. 2010, A\&A, 518, A6 Barklem, P. S., \& O'Mara, B. J. 1997, MNRAS, 290, 102
Barklem, P. S., O’Mara, B. J., \& Ross, J. E. 1998, MNRAS, 296, 1057

Beck, C., Rezaei, R., \& Fabbian, D. 2011, A\&A, 535, A129

Bello González, N., \& Kneer, F. 2008, A\&A, 480, 265

Bellot Rubio, L. R., Collados, M., Ruiz Cobo, B., \& Rodríguez Hidalgo, I. 2000, ApJ, 534, 989

Bellot Rubio, L. R., Balthasar, H., \& Collados, M. 2004, A\&A, 427, 319

Berkefeld, T., Schmidt, D., Soltau, D., von der Lühe, O., \& Heidecke, F. 2012, Astron. Nachr., 333, 863

Bharti, L., Cameron, R. H., Rempel, M., Hirzberger, J., \& Solanki, S. K. 2012, ApJ, 752, 128

Bianda, M., Solanki, S. K., \& Stenflo, J. O. 1998, A\&A, 331, 760

Bloomfield, D. S., Solanki, S. K., Lagg, A., Borrero, J. M., \& Cally, P. S. 2007, A\&A, 469, 1155

Borrero, J. M., \& Bellot Rubio, L. R. 2002, A\&A, 385, 1056

Borrero, J. M., \& Ichimoto, K. 2011, Liv. Rev. Sol. Phys., 8, 4

Borrero, J. M., \& Solanki, S. K. 2008, ApJ, 687, 668

Borrero, J. M., \& Solanki, S. K. 2010, ApJ, 709, 349

Borrero, J. M., Bellot Rubio, L. R., Barklem, P. S., \& del Toro Iniesta, J. C. 2003, A\&A, 404, 749

Borrero, J. M., Solanki, S. K., Bellot Rubio, L. R., Lagg, A., \& Mathew, S. K. 2004, A\&A, 422, 1093

Borrero, J. M., Lagg, A., Solanki, S. K., \& Collados, M. 2005, A\&A, 436, 333

Borrero, J. M., Solanki, S. K., Lagg, A., Socas-Navarro, H., \& Lites, B. 2006, A\&A, 450, 383

Borrero, J. M., Lites, B. W., \& Solanki, S. K. 2008, A\&A, 481, L13

Borrero, J. M., Lites, B. W., Lagg, A., Rezaei, R., \& Rempel, M. 2014, A\&A, 572, A54

Cabrera Solana, D., Bellot Rubio, L. R., Beck, C., \& Del Toro Iniesta, J. C. 2007, A\&A, 475, 1067

Cabrera Solana, D., Bellot Rubio, L. R., Borrero, J. M., \& Del Toro Iniesta, J. C. 2008, A\&A, 477, 273

Casini, R., Asensio Ramos, A., Lites, B. W., \& López Ariste, A. 2013, ApJ, 773, 180

Chandrasekhar, S., \& Breen, F. H. 1946, ApJ, 104, 430

Collados, M. 2007, in Modern solar facilities - advanced solar science, Proc. of a Workshop held at Göttingen September 27-29, 2006, eds. F. Kneer, K. G. Puschmann, \& A. D. Wittmann, 143

Collados, M., Martinez Pillet, V., Ruiz Cobo, B., del Toro Iniesta, J. C., \& Vazquez, M. 1994, A\&A, 291, 622

Collados, M., López, R., Páez, E., et al. 2012, Astron. Nachr., 333, 872

Danilovic, S., Gandorfer, A., Lagg, A., et al. 2008, A\&A, 484, L17

Del Moro, D., Stangalini, M., Viticchiè, B., et al. 2010, Mem. Soc. Astron. It. Suppl., 14, 180

del Toro Iniesta, J. C. 2003, Introduction to Spectropolarimetry (Cambridge, UK: Cambridge University Press)

del Toro Iniesta, J. C., Bellot Rubio, L. R., \& Collados, M. 2001, ApJ, 549, L139 Esteban Pozuelo, S., Bellot Rubio, L. R., \& de la Cruz Rodríguez, J. 2015, ApJ, 803,93

Franz, M., \& Schlichenmaier, R. 2009, A\&A, 508, 1453

Franz, M., \& Schlichenmaier, R. 2013, A\&A, 550, A97

Franz, M., Collados, M., \& Bethge, C. et. al. 2016, A\&A, 596, A4 (GREGOR SI)

Frutiger, C., Solanki, S. K., Fligge, M., \& Bruls, J. H. M. J. 1999, in Polarization, eds. K. N. Nagendra \& J. O. Stenflo, Astrophys. Space Sci. Lib., 243, 281

Golub, G., \& Kahan, W. 1965, SIAM J. Num. Anal., 2, 205

Ichimoto, K. 2010, in Magnetic Coupling between the Interior and Atmosphere of the Sun, eds. S. S. Hasan, \& R. J. Rutten, 186

Jahn, K., \& Schmidt, H. U. 1994, A\&A, 290, 295

Joshi, J., Pietarila, A., Hirzberger, J., et al. 2011, ApJ, 734, L18

Keller, C. U., \& von der Luehe, O. 1992, A\&A, 261, 321

Lagg, A., Solanki, S. K., van Noort, M., \& Danilovic, S. 2014, A\&A, 568, A60

Lites, B. W., Elmore, D. F., Seagraves, P., \& Skumanich, A. P. 1993, ApJ, 418, 928

Livingston, W., \& Wallace, L. 1991, N.S.O. Tech. Rep., 91, 001

Löfdahl, M. G. 2002, in Image Reconstruction from Incomplete Data, eds. P. J. Bones, M. A. Fiddy, \& R. P. Millane, SPIE Conf. Ser., 4792, 146

Löfdahl, M. G., \& Scharmer, G. B. 2012, A\&A, 537, A80

Lucy, L. B. 1974, AJ, 79, 745

Martínez Pillet, V., Del Toro Iniesta, J. C., et al. 2011, Sol. Phys., 268, 57

Mathew, S. K., Lagg, A., Solanki, S. K., et al. 2003, A\&A, 410, 695

Mathew, S. K., Zakharov, V., \& Solanki, S. K. 2009, A\&A, 501, L19

Nave, G., Johansson, S., Learner, R. C. M., Thorne, A. P., \& Brault, J. W. 1994, ApJS, 94, 221

Nordlund, A., \& Scharmer, G. B. 2010, Astrophys. Space Sci. Proc., 19, 243

Paxman, R. G., Seldin, J. H., Loefdahl, M. G., Scharmer, G. B., \& Keller, C. U. 1996, ApJ, 466, 1087

Press, W. H., Flannery, B. P., \& Teukolsky, S. A. 1986, Numerical recipes. The art of scientific computing (Cambridge University Press) 
Puschmann, K. G., Ruiz Cobo, B., \& Martínez Pillet, V. 2010, ApJ, 720, 1417 Quintero Noda, C., Asensio Ramos, A., Orozco Suárez, D., \& Ruiz Cobo, B. 2015, A\&A, 579, A3

Rempel, M. 2011, ApJ, 729, 5

Rempel, M. 2012, ApJ, 750, 62

Rempel, M., Schüssler, M., Cameron, R. H., \& Knölker, M. 2009, Science, 325, 171

Richardson, W. H. 1972, J. Opt. Soc. Am., 62, 55

Riethmüller, T. L., Solanki, S. K., van Noort, M., \& Tiwari, S. K. 2013, A\&A, 554, A53

Ruiz Cobo, B., \& Asensio Ramos, A. 2013, A\&A, 549, L4

Ruiz Cobo, B., \& del Toro Iniesta, J. C. 1992, ApJ, 398, 375

Scharmer, G. B. 2008, Phys. Scr. T, 133, 014015

Scharmer, G. B., \& Henriques, V. M. J. 2012, A\&A, 540, A19

Scharmer, G. B., \& Spruit, H. C. 2006, A\&A, 460, 605

Scharmer, G. B., Narayan, G., Hillberg, T., et al. 2008, ApJ, 689, L69

Scharmer, G. B., Henriques, V. M. J., Kiselman, D., \& de la Cruz Rodríguez, J. 2011, Science, 333, 316

Scharmer, G. B., de la Cruz Rodriguez, J., Sütterlin, P., \& Henriques, V. M. J. 2013, A\&A, 553, A63

Schlichenmaier, R., \& Franz, M. 2013, A\&A, 555, A84
Schmidt, W., von der Lühe, O., Volkmer, R., et al. 2012, Astron. Nachr., 333, 796

Skumanich, A., \& López Ariste, A. 2002, ApJ, 570, 379

Socas-Navarro, H., de la Cruz Rodríguez, J., Asensio Ramos, A., Trujillo Bueno, J., \& Ruiz Cobo, B. 2015, A\&A, 577, A7

Solanki, S. K., \& Montavon, C. A. P. 1993, A\&A, 275, 283

Spruit, H. C., \& Scharmer, G. B. 2006, A\&A, 447, 343

Spruit, H. C., Scharmer, G. B., \& Löfdahl, M. G. 2010, A\&A, 521, A72

Suematsu, Y., Tsuneta, S., Ichimoto, K., et al. 2008, Sol. Phys., 249, 197

Tiwari, S. K., van Noort, M., Lagg, A., \& Solanki, S. K. 2013, A\&A, 557, A25

Tiwari, S. K., van Noort, M., Solanki, S. K., \& Lagg, A. 2015, A\&A, 583, A119 van Noort, M. 2012, A\&A, 548, A5

van Noort, M., Rouppe van der Voort, L., \& Löfdahl, M. G. 2005, Sol. Phys., 228, 191

van Noort, M., Lagg, A., Tiwari, S. K., \& Solanki, S. K. 2013, A\&A, 557, A24

Volkmer, R., Eisenträger, P., Emde, P., et al. 2012, Astron. Nachr., 333, 816 Wedemeyer-Böhm, S. 2008, A\&A, 487, 399

Yeo, K. L., Feller, A., Solanki, S. K., et al. 2014, A\&A, 561, A22

Zakharov, V., Hirzberger, J., Riethmüller, T. L., Solanki, S. K., \& Kobel, P. 2008, A\&A, 488, L17 